\title{
Fully-implicit simulation of vertical-equilibrium models with hysteresis and capillary fringe
}

\author{
Halvor Møll Nilsen ${ }^{1} \cdot$ Knut-Andreas Lie $^{1} \cdot$ Odd Andersen $^{1,2}$ (D)
}

Received: 1 October 2014 / Accepted: 27 October 2015 / Published online: 26 November 2015

(C) The Author(s) 2015. This article is published with open access at Springerlink.com

\begin{abstract}
Geological carbon storage represents a new and substantial challenge for the subsurface geosciences. To increase understanding and make good engineering decisions, containment processes and large-scale storage operations must be simulated in a thousand year perspective. A hierarchy of models of increasing computational complexity for analysis and simulation of large-scale $\mathrm{CO}_{2}$ storage has been implemented as a separate module of the opensource Matlab Reservoir Simulation Toolbox (MRST). This paper describes a general family of two-scale models available in this module. The models consist of two-dimensional flow equations formulated in terms of effective quantities obtained from hydrostatic reconstructions of vertical pressure and saturation distributions. The corresponding formulation is fully implicit and is the first to give a massconservative treatment and include general (non-linearized) $\mathrm{CO}_{2}$ properties. In particular, the models account for compressibility, dissolution, and hysteresis effects in the finescale capillary and relative permeability functions and can be used to accurately and efficiently study the combined large-scale and long-term effects of structural, residual, and solubility trapping.
\end{abstract}

Odd Andersen

odd.andersen@sintef.no

Halvor Møll Nilsen

halvor.m.nilsen@sintef.no

Knut-Andreas Lie

knut-andreas.lie@sintef.no

1 Department of Applied Mathematics, SINTEF ICT, P.O. Box 124 Blindern, N-0314 Oslo, Norway

2 Department of Mathematics, University of Bergen, Bergen, Norway
Keywords $\mathrm{CO} 2$ storage · Vertical equilibrium · Capillary fringe $\cdot$ Hysteresis

\section{Introduction}

Carbon capture and storage (CCS), which refers to the capture of $\mathrm{CO}_{2}$ from large point sources in power generation and industry followed by permanent storage, is one strategy for reducing emissions contributing to climate change. Geological sequestration provides an option for permanent storage of $\mathrm{CO}_{2}$ at an industrial scale. At present, it is widely accepted that geological carbon storage is possible and can be conducted with reasonable safety for moderately large injection volumes. Nevertheless, if geological storage is to make a significant impact on global emissions, storage operations must be scaled up to a level that involves the injection of gigatonnes of $\mathrm{CO}_{2}$ per year. $\mathrm{CO}_{2}$ can potentially be stored in depleted oil or gas fields, unmineable coal seams, and saline aquifers. Herein, we consider large-scale utilization of saline aquifers to store gigatonnes of $\mathrm{CO}_{2}$.

Before a saline aquifer can be used for large-scale carbon storage, any operator, investor, or governmental agency taking an environmental, societal, or financial risk in a storage operation needs to perform extensive mapping, analysis, and planning to determine its operational performance, financial costs, and long-term security. The only viable way to make such assessments upfront is through computerized model studies that aim to forecast the likely outcomes of the storage operation. This, in turn, requires a good understanding of the injection and migration processes, which are governed by a number of physical mechanisms involving multiple spatial and temporal scales. Analysis and planning will take place in a number of stages that each will require different models and computational methods. During the initial 
screening and early injection planning, one primarily needs tools for studying the effects of various trapping mechanisms and determining large-scale storage capacity and injection properties. The large degree of uncertainty inherent in the input parameters (aquifer geometry, petrophysical properties, boundary conditions and pressure regimes, fluid properties, etc.) requires flexible and computationally inexpensive tools that can quickly be turned around to study alternative model assumptions and gradually be refined to account for increasingly complex description of the aquifer geology and flow physics. The tools should also be formulated so that they can use mathematical optimization methods to develop optimal injection scenarios. As one approaches detailed injection planning and the operational phase, one will need to use advanced 3D multiscale multiphysics simulators to study the local and short-term interplay of pressure buildup and multiphase, geomechanical, thermal, and geochemical effects. Likewise, as injection progresses, one will need tools that can perform continuous integration of data and eventually function alongside with instrumentation for long-term monitoring of the injected $\mathrm{CO}_{2}$ plumes.

Experience has shown that storage sites also vary a lot in operational constraints and physical characteristics [1]. One therefore needs a toolchain of interoperable mathematical models and computational methods so that computer modeling can be adapted on a case-by-case basis to account for the salient physical effects at a level of accuracy that is commensurate with the available data. As a step in this direction, we have developed MRST-CO2 lab [2], which is an add-on module to the open-source Matlab Reservoir Simulation Toolbox (MRST) [3-6]. Through its various add-on modules, MRST offers capabilities for industry-standard reservoir simulation as well as a number of multiscale and multiphysics capabilities that can be used to perform detailed 3D simulation of $\mathrm{CO}_{2}$ injection on a reservoir scale. The purpose of MRST-CO2lab, on the other hand, is to provide functionality for modeling and simulation of $\mathrm{CO}_{2}$ storage in a long-term, large-scale perspective. To this end, the software offers two kind of computational tools: (i) methods that rapidly identify spill paths and estimate upper bounds on structural, residual, and solubility trapping without the use of temporal information [7] and (ii) methods that forecast the likely outcomes of injection operations in a long-term, large-scale perspective by simulating the combined effects of structural, residual, and solubility trapping in a vertically averaged sense assuming a vertical equilibrium. The purpose of the current paper and its companion [8] is to describe the latter simulation capabilities in detail. In a series of related papers [9-11], we combine the tools from MRST-CO2lab to estimate storage capacities using data sets from the recent $\mathrm{CO}_{2}$ Storage Atlas of the Norwegian Continental Shelf [12], which describe large-scale saline aquifer systems, as well as more localized models describing the Sleipner injection site and parts of the Johansen formation. In particular, by combining the fast simulation capabilities with mathematical optimization methods, we develop efficient workflows that can be used to suggest placement of storage hubs and optimized injection strategies for large-scale utilization of basin-scale aquifer systems. Finally, we mention that work is in progress to include additional physics like thermal, geochemical, and geomechanical effects in MRST - $\mathrm{CO} 2 \mathrm{lab}$.

\section{Simulating $\mathrm{CO}_{2}$ migration in large-scale aquifer systems}

In most realistic cases, the $\mathrm{CO}_{2}$ injected into a saline aquifer will be in a supercritical phase that is less dense than the surrounding brine and will therefore form a distinct plume that tends to migrate upwards unless held back by one or more trapping mechanisms. The migration process will only cease when all the injected $\mathrm{CO}_{2}$ has been permanently immobilized by trapping mechanisms or otherwise leaked back out.

As has been clearly demonstrated in benchmark studies $[13,14]$, it is challenging, if possible at all, to use stateof-the-art methods for 3D simulation to provide spatially resolved simulation of the gravity-controlled migration and trapping of a buoyant $\mathrm{CO}_{2}$ phase beneath caprock barriers under near-hydrostatic conditions. Moreover, this is a long-term process that can go on for millennia in large sloping aquifer systems. Even for conceptual models with very simple physics, 3D simulators tend to underestimate migration velocities by more than $30 \%$ [14]. This is caused by a disparity in physical scales: Whereas the aquifers themselves may be thousands of square kilometers in areal extent the formation height will typically be a couple of hundreds of meters or less. Gravity-dominated flow during the long migration period leads to the formation of a very thin $\mathrm{CO}_{2}$ plume, which means that the important trapping and migration processes take place on a meter scale or less. High resolution in the vertical direction is therefore required to resolve the gravity segregation inside of the plume, whose upward migration is sensitive to small undulations in the caprock topography that may both retard the migration of the plume and divert its path. Likewise, high grid resolution is required in the lateral direction to resolve the unstable dynamics of convective dissolution. Dissolution retards and limits the extent of the plume migration and can increase the storage capacity by a factor two or more compared to what is attainable by structural trapping alone. Looking at time scales, one observes that because of the large difference in density between the supercritical $\mathrm{CO}_{2}$ plume and the resident brine and the fact that the aquifer can be viewed 
as a thin, slightly inclined sheet, the segregation of fluids can be considered almost instantaneous compared with the lateral movement of the $\mathrm{CO}_{2}$ plume. When studying plume migration in a thousand-year perspective, it is therefore reasonable to assume that the fluids at all times are segregated and in vertical equilibrium, see, e.g., [15].

Another argument against the use of traditional 3D simulators to model large-scale, long-term migration of $\mathrm{CO}_{2}$ in large aquifer systems is the lack of reliable data describing the rock formations. It is well known that geological heterogeneity strongly impacts the movement of fluids in subsurface rock formations regardless of whether the fluid movement is driven by viscous forces on a reservoir scale or by buoyancy forces on a basin scale. However, although saline aquifers have a complex layered structure, detailed modeling of this structure is not supported by available data: Seismic surveys have limited coverage, and there are few exploration wells that can provide core samples. Geological models therefore tend to be relatively homogeneous and caprock topography is in many cases the primary geological parameter that determines long-term $\mathrm{CO}_{2}$ migration. This is particularly true for the public data sets that describe the enormous volumes of potential storage capacity on the Norwegian Continental Shelf, see, e.g., [12], which is the main motivation for our work.

With a vertical equilibrium assumption, the flow of a thin $\mathrm{CO}_{2}$ plume can be approximated in terms of its thickness to obtain a 2D depth-integrated simulation model. This type of semi-analytical approach has long traditions in different branches of physics. One example is the shallow-water or Saint Venant equations that describe the flow under a free surface. Another example is the Dupuit approximation in hydrology from 1863, which states that if the water table in an unconfined aquifer is relatively flat and the groundwater is hydrostatic, then the water discharge is proportional to the thickness of the saturated aquifer [16]. In the petroleum industry, similar models were developed more than 50 years ago to study segregated flow [17-20]. In recent years, vertical-equilibrium (VE) models have been extensively used to study gravity-driven $\mathrm{CO}_{2}$ migration, see [15], and the validity of the assumptions lading up to VE models has been investigated with respect to spatial [21] and temporal [22] scales. Early methods assumed a sharp interface between $\mathrm{CO}_{2}$ and the resident brine and were successfully applied to study long-term $\mathrm{CO}_{2}$ migration in the large-scale Utsira [23] and Johansen [24] aquifers. Since then, the VE formulation has been extended to include many relevant physical effects, such as compressibility [25], convective dissolution [26], capillary fringe [27], smallscale caprock topography variations [28-30], as well as various hysteretic effects [31, 32]. Presently, VE formulations can be used to efficiently simulate $\mathrm{CO}_{2}$ migration and resolve the delicate balances governing structural, residual, and solubility trapping over thousand of years. Using an analytical description for the vertical fluid distribution not only reduces the dimensions of the problem but also lessens the coupling and increases the time constants of the dynamic model. Moreover, important information of the heterogeneities in the underlying 3D medium is preserved, and the errors introduced by assuming vertical equilibrium are in many cases significantly smaller than the errors induced by the overly coarse resolution needed to make a 3D simulation computationally tractable [33]. Recently, it was also demonstrated how one can efficiently simulate vertical-equilibrium models even when the vertical integrals for efficient properties cannot be approximated by closedform expressions and must be integrated on the fly [34]. Altogether, extensive numerical verification and validation shows that vertically-integrated models in general, and vertical equilibrium models in particular, are attractive means to increase resolution while saving computational cost. For the Sleipner injection, for instance, it has been shown that vertical-equilibrium models give equally good match, if not better, than commercial and research simulators based on traditional 3D discretizations [23, 35, 36].

Through MRST-CO2lab, we have tried to develop an open-source framework for vertically-integrated modeling of $\mathrm{CO}_{2}$ that includes the majority of the model features reported in the literature, including in particular fine-scale and upscaled capillary effects, convective dissolution, finescale and upscaled hysteretic behavior, vertical heterogeneity in lateral permeability, as well as retardation effects of unresolved caprock variations. Vertically-integrated models have the same structure as traditional equations for multiphase flow in porous media. In fact, although this is not common in the literature, these models can be formulated in the black-oil framework which is the industry standard for simulating petroleum reservoirs. Several commercial reservoir simulators have vertical-equilibrium options that work with both fully-implicit and sequential solution strategies, but to the best of our knowledge, neither of these simulators currently support recent features developed to study the combined large-scale and long-term effects of structural, residual, and solubility trapping of $\mathrm{CO}_{2}$. On the other hand, most research codes used in the literature to study $\mathrm{CO}_{2}$ sequestration are based on simple IMPES or sequential-splitting methods that generally lack the robustness of fully-implicit solvers. In particular, the VESA code [37], which to the best of our knowledge is the most general code reported in the literature, uses a nonstandard nonconservative version of IMPES, but nevertheless seems to work rather well. However, like most other research codes, it is not available to the public and thus cannot be used for reproducible research.

To ensure that the models can be seamlessly integrated with standard models used for 3D simulation and easily 
be implemented in commercial reservoir simulators, all VE models in MRST-CO2lab, except for very simple sharpinterface models, are formulated in a black-oil type framework. Likewise, for the numerical discretization, we use standard solution methods developed over the last decades within petroleum reservoir simulation. Apart from adapting to the industry-standard in petroleum engineering, our main motivation for using such fully-implicit methods is that they are particularly robust and efficient for transient simulations approaching a steady state, which will be the case when $\mathrm{CO}_{2}$ is trapped, as discussed in more detail in [8]. An attractive feature of MRST is that it greatly simplifies the development of fully implicit simulators for complex, coupled systems through a combination of abstract discretization operators and algorithmic differentiation for automatic computation of Jacobians [5, 6]. This greatly simplifies the task of computing gradients and parameter sensitivities using an adjoint formulation and extend the VE models with new features like capillary and hysteretic effects. Apart from a rapid development cycle in MATLAB compared with compiled languages, the main purpose of using MRST is to make the new methods interoperable with existing functionality. A wide range of traditional 3D models and simulators is already implemented in the software, including standard black-oil models and certain EOR models, as well as thermal, geochemical, and simple mechanical effects. MRST also provides efficient preconditioners and multigrid solvers as well as adjoint methods for efficient optimization.

In [8], we discuss vertical-equilibrium models for simulating structural, residual, and solubility trapping based on an assumption of a sharp interface between the injected $\mathrm{CO}_{2}$ and formation water. These models are highly computationally efficient but also have important limitations, such as a tendency of overestimating the velocity of the tip of the $\mathrm{CO}_{2}$ plume [27]. In the current paper, we present a more comprehensive framework for VE models that also takes into account fine-scale capillary pressure effects that will create capillary fringes, i.e., smooth transitions between pure $\mathrm{CO}_{2}$ and pure brine. We discuss in detail the modeling of hysteretic behavior and its impact on the computed effective quantities and present a number of numerical examples that compare various approximations that can be made to simplify the mathematical models and speed up the computations. To keep the presentation as simple as possible, we only consider 2D aquifer cross sections which translate to $1 \mathrm{D}$ VE models. Examples of more comprehensive simulation on real 3D models can be found in [9-11, 38, 39].

\section{Basic VE models with a capillary fringe}

We consider the evolution of a $\mathrm{CO}_{2}$ plume in a tilted aquifer. As time goes by, structural, residual, and solubility trapping will separate the injected $\mathrm{CO}_{2}$ into different forms as illustrated in Fig. 1.

In the figure, $\mathrm{CO}_{2}$ and brine are assumed to be separated by a sharp interface, leading to a fluid distribution consisting of five zones: $\mathrm{CO}_{2}$ confined in structural traps, free flowing $\mathrm{CO}_{2}$, brine with residual $\mathrm{CO}_{2}$, brine with dissolved $\mathrm{CO}_{2}$, and pure brine. More details about sharpinterface models and the carbon inventory as provided by MRST-CO2lab is given in [8]. For models with a capillary fringe, an approximate inventory can be computed by reinterpreting the vertical fluid distribution in terms of a sharp-interface model.

The assumption of a sharp interface is reasonable when fluid saturations go from minimum to maximum values
Fig. 1 Schematic of a vertical section of an aquifer showing the different forms in which the injected $\mathrm{CO}_{2}$ can be present during migration. The right inlet shows small-scale undulations in the top surface which typically will not be resolved in a largescale model. The left inlet shows a detailed inventory of various categories of trapped $\mathrm{CO}_{2}$, stacked in terms of increasing leakage risk, as function of time from the end of injection

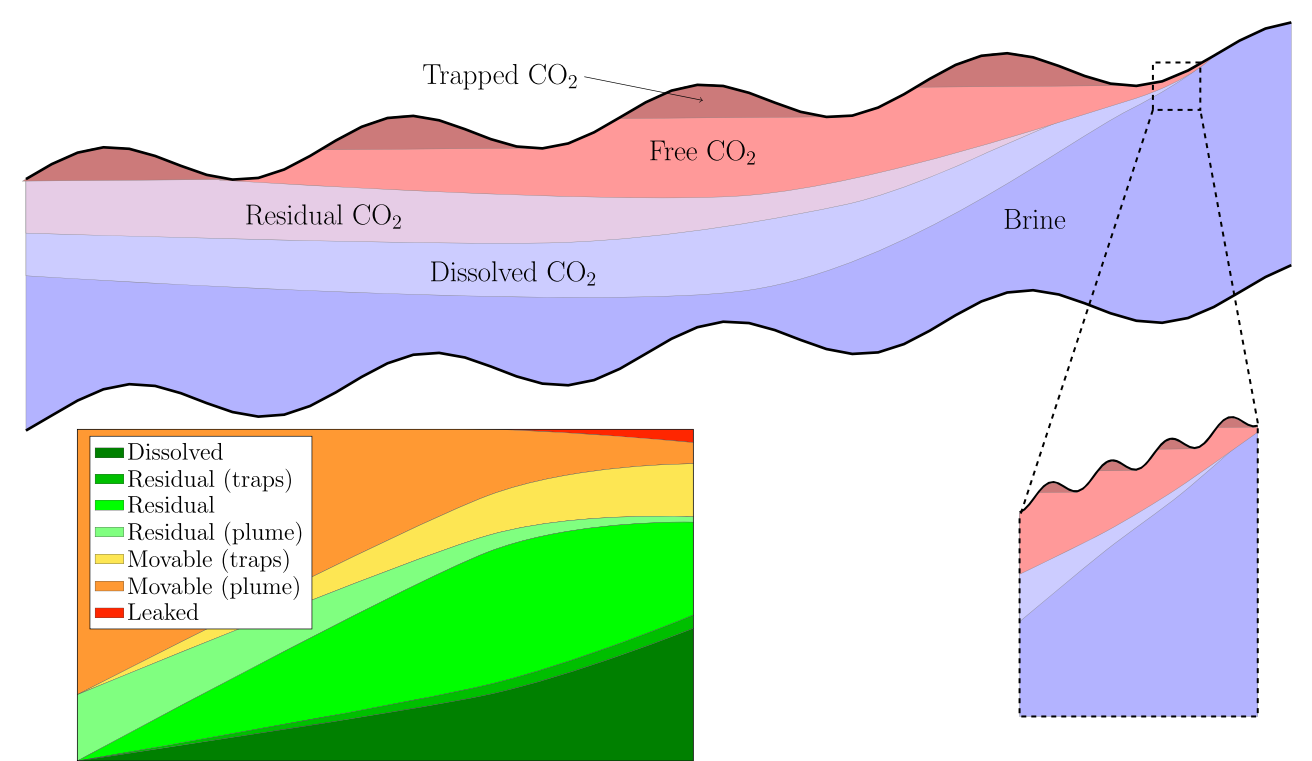


for a change in capillary pressure that is negligible compared with the change in pressures across the whole local thickness of the aquifer. However, if this is not the case, a capillary fringe will develop, within which saturations will vary gradually orthogonal to the main flow direction. The main large-scale effect of introducing a capillary fringe is reduced effective $\mathrm{CO}_{2}$ mobility for small plume heights, which slows down the advancement of the plume front and introduces a rarefaction at the trailing edge. The effect is qualitatively similar to the retardation effect induced by small-scale caprock undulations, which is discussed in detail in [8].

The starting point for our framework is the standard equations for a two-phase flow system on the fine scale,

$$
\begin{gathered}
\frac{\partial}{\partial t}\left(\phi s_{\alpha}\right)+\nabla \cdot \vec{v}_{\alpha}=q_{\alpha}, \\
\vec{v}_{\alpha}=-\mathbf{k} \lambda_{\alpha}\left(\nabla p_{\alpha}-\rho_{\alpha} \vec{g}\right) .
\end{gathered}
$$

Here, brine and $\mathrm{CO}_{2}$ are modeled as wetting and nonwetting fluids, respectively, $\phi$ denotes porosity, $\mathbf{k}$ permeability, and $\vec{g}$ the gravity vector, whereas $p_{\alpha}, s_{\alpha}, \lambda_{\alpha}$, and $q_{\alpha}$ denote pressure, saturation, mobility, and volumetric source for phase $\alpha=\{w, n\}$. For simplicity, we limit our discussion in this section to cases without hysteresis, i.e., we assume no residual $\mathrm{CO}_{2}$ in the brine zone, which is a valid assumption in the advancement (drainage) zone of the $\mathrm{CO}_{2}$ plume; we will return to models with hysteresis in Section 5. Likewise, the model equations include rock compressibility but disregard fluid compressibility. The effect of variable density in vertical-equilibrium models was investigated by Andersen et al. [25], who concluded that for the vast majority of long-term, large-scale storage systems it is sufficient to account for $\mathrm{CO}_{2}$ density variations only in the lateral direction (i.e., a semi-compressible model). Both fully compressible and semi-compressible models have been implemented in MRST, but for brevity we herein disregard fluid compressibility when discussing how to derive expressions for effective properties by vertical integration.

As in [8], we introduce a curvilinear coordinate system $\left(\vec{e}_{\xi}, \vec{e}_{\eta}, \vec{e}_{\zeta}\right)$ that follows the main directions of flow as illustrated in Fig. 2. In the following, subscript $\|$ refers to the $\left(\vec{e}_{\xi}, \vec{e}_{\eta}\right)$ components of a vector or operator. The new coordinate system is discussed in more detail in [8].

Integrating (1) from top to bottom of the aquifer (and neglecting distortions from the curvilinear nature of the coordinate system [8]), gives:

$$
\frac{\partial}{\partial t}\left[\int_{Z}^{Z+H} s_{\alpha} \phi d \zeta\right]+\nabla_{\|} \cdot\left[\int_{Z}^{Z+H} \vec{v}_{\alpha} d \zeta\right]=\int_{Z}^{Z+H} q_{\alpha} d \zeta
$$

The flow component along $\vec{e}_{\zeta}$ is zero or negligible, and we therefore assume that the phase pressures $p_{n}$ and $p_{w}$ are in hydrostatic equilibrium along this direction (which we henceforth will refer to as 'vertical'). Herein, we will make the simplifying assumption that the density is constant within each vertical column, which is reasonable in most cases in which VE-models are suited for modeling $\mathrm{CO}_{2}$ storage [25]. Moreover, to simplify the notation, we will integrate from 0 to $H$ instead of from $Z$ to $Z+H$ when considering a single column. Integrating the left two terms of Eq. 3 requires treating fine-scale phase saturation and pressure as functions of depth. If we let $p_{w, i}$ and $p_{n, i}$ denote the phase pressures at the interface between $\mathrm{CO}_{2}$ and water, defined as the maximum depth $h$ at which mobile $\mathrm{CO}_{2}$ is present, we can then determine the capillary pressure $p_{c}$ as a function of depth according to the following formula:

$$
\begin{aligned}
p_{w}(z ; h) & =p_{w, i}+g \rho_{w}(z-h), \\
p_{\mathrm{n}}(z ; h) & =p_{\mathrm{n}, i}+g \rho_{\mathrm{n}}(z-h), \\
p_{c}(z ; h) & =p_{c, i}+g \Delta \rho(z-h) .
\end{aligned}
$$

Above, $\Delta \rho=\rho_{\mathrm{n}}-\rho_{w}$ is the density difference and $p_{c, i}=$ $p_{\mathrm{n}, i}-p_{w, i}$ is the capillary pressure at $z=h$, which is the capillary pressure necessary to initiate displacement of brine by $\mathrm{CO}_{2}$, also called the entry pressure. In the case

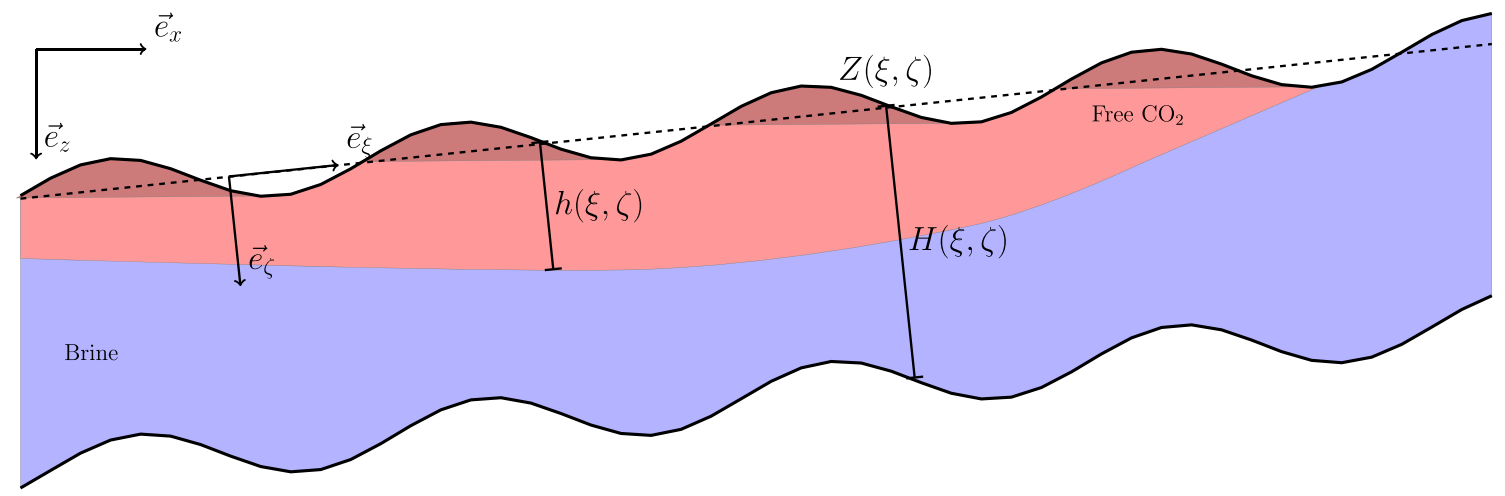

Fig. 2 Schematic of the coordinate systems used to derive verticalequilibrium models. The dashed line indicates main assumed direction of flow within the aquifer, which is here illustrated as straight, but is allowed to be slightly curved in the general case. The aquifer is described by the top surface $Z(\xi, \eta)$ and its thickness $H(\xi, \eta)$ in the $\zeta$-direction 
Fig. 3 Diagrams showing phase pressures (left) and brine saturation (right) as functions of depth $z$. In the left plot, the capillary pressures at depths $h$ and $h_{m}$ are indicated. The zone where saturation varies with depth is identified as the capillary fringe on the right plot
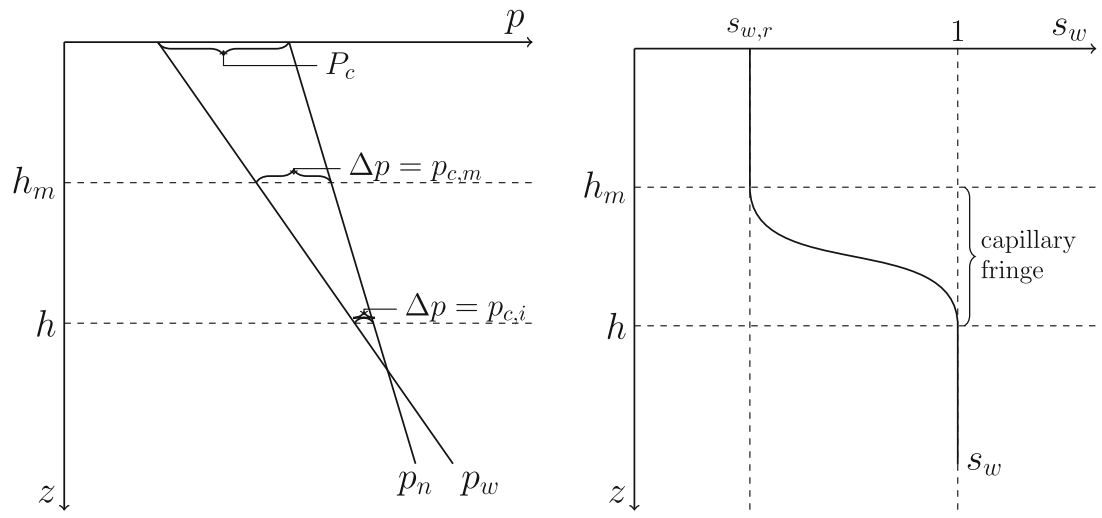

without hysteresis, $h$ is equivalent to the maximum depth with nonzero $\mathrm{CO}_{2}$ saturation $s_{n}$. The formula (4) assumes constant vertical density, but an extension of the VE formulation without this assumption is studied in [25], in which case the vertical pressure profile is reconstructed by solving an ordinary differential equation.

The left diagram in Fig. 3 illustrates the hydrostatic pressure profiles of $\mathrm{CO}_{2}$ and brine and identifies the entry pressure $p_{c, i}$ as well as the capillary pressure value $p_{c, m}$, above which brine stops flowing entirely. The diagram also identifies the capillary pressure at the top surface, $P_{c}$, which is sometimes interpreted as the upscaled capillary pressure. The corresponding brine saturation is illustrated in the right diagram, where we see that it varies smoothly between $h_{m}$ and $h$ (within the capillary fringe) and assumes constant values elsewhere.

From the expression of fine-scale capillary pressure, and assuming a given, monotone function $\hat{p}_{c}\left(s_{w}\right)$ relating capillary pressure to brine saturation (henceforth, a hat will denote an input function given either as an analytic expression or in tabulated form), we can now reconstruct the fine-scale saturation in the region where $\hat{p}_{c}^{-1}$ exists:

$s_{w}(z)= \begin{cases}s_{w, r}=\hat{p}_{c}^{-1}\left(p_{c, m}\right), & z \leq h_{m} \\ \hat{p}_{c}^{-1}\left(p_{c, i}+g \Delta \rho(z-h)\right), & h_{m} \leq z \leq h, \\ 1=\hat{p}_{c}^{-1}\left(p_{c, i}\right), & h \leq z .\end{cases}$

Given this function, we can calculate the upscaled quantities:

$$
\begin{aligned}
S_{\mathrm{n}} & =\frac{1}{\Phi H} \int_{0}^{H}\left[1-s_{w}(z)\right] \phi(z) \mathrm{d} z, \\
\Lambda_{\mathrm{n}} & =\frac{1}{H}\left[\int_{0}^{H} \mathbf{k}_{\|}(z) \lambda_{\mathrm{n}}\left(1-s_{w}(z)\right) \mathrm{d} z\right] \mathbf{K}^{-1}, \\
\Lambda_{w} & =\frac{1}{H}\left[\int_{0}^{H} \mathbf{k}_{\|}(z) \lambda_{w}\left(s_{w}(z)\right) \mathrm{dz}\right] \mathbf{K}^{-1},
\end{aligned}
$$

where the petrophysical properties have been upscaled as follows:

$$
\Phi=\frac{1}{H} \int_{0}^{H} \phi d \zeta, \quad \mathbf{K}=\frac{1}{H} \int_{0}^{H} \mathbf{k}_{\|} d \zeta .
$$

Whereas (5) here has been derived from the incompressible model described by Eqs. 1-2, the definitions remain the same also for a model with compressible fluids. In the rest of the paper, we focus on the evaluation of effective properties in cases where density can be approximated as constant within each vertical column, i.e., as described by the semi-compressible model of [25].

We are now in a position to formulate the upscaled flow equations, which can be developed as explained in [8] using either $h$ or $S_{n}$ as the primary variable. In addition, the coarse-scale pressure $P$ is taken as the water pressure at the top of the aquifer, $p_{w}(0)=p_{w, i}-g h \rho_{w}$, and this is the pressure we will use when evaluating densities henceforth. Implications of the choice of independent variables will be discussed in more details in the next section.

\section{Evaluation of effective properties}

A main choice distinguishing different VE models is how to reconstruct the saturation profile and evaluate the effective parameters of the upscaled flow equations based on this reconstruction. The full tree of dependencies among finescale and upscaled variables is summarized in Fig. 4. In this

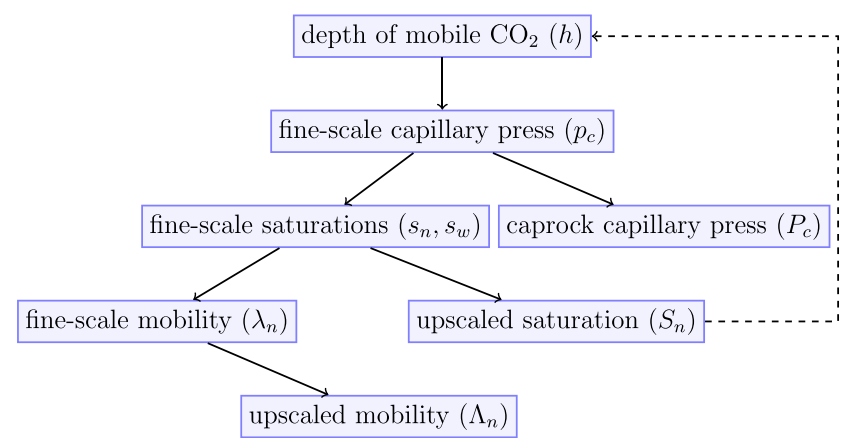

Fig. 4 Dependence among fine-scale and upscaled parameter in a general VE model with capillary fringe. If $S_{n}$ is the independent variable, then $h$ must first be computed by inverting $S_{n}(h)$ before one can reconstruct fine-scale fluid distribution and compute upscaled quantities 
Table 1 The choice of independent variables and the shape of the vertically-averaged relative-permeability and capillary-pressure functions are affected by three factors in the underlying 3D model: vertical variations in permeability and porosity, capillary pressure, and compressibility

\begin{tabular}{lllllll}
\hline Model type & & & $k_{r}$ & & $p_{c}$ & \\
\hline rock parameters & capillary & compressible & variables & feature & variables & feature \\
constant & no & no & $S$ & linear & $S$ & linear \\
varying $\mathbf{k}$ & no & no & $S$ & nonlinear & $S$ & linear \\
varying $\phi$ & no & no & $S$ & nonlinear & $S$ & nonlinear \\
constant & no & yes & $S$ & nonlinear & $\boldsymbol{S}, \boldsymbol{P}$ & nonlinear \\
constant & yes & no & $S$ & nonlinear & $S$ & nonlinear \\
constant & yes & yes & $\boldsymbol{S}, \boldsymbol{P}$ & nonlinear & $\boldsymbol{S}, \boldsymbol{P}$ & nonlinear \\
varying $\phi$ & no & yes & $\boldsymbol{S}, \boldsymbol{P}$ & nonlinear & $\boldsymbol{S}, \boldsymbol{P}$ & nonlinear \\
\hline
\end{tabular}

section, we look at how the choice of primary variables as well as the physical effects included in the model impact the computation of effective properties and compared the pros and cons of the $S$-formulation and the $h$-formulations from a numerical point-of-view.

To prepare for this discussion, we will first look at new functional dependencies that appear in the effective properties in general VE models. For constant rock parameters, incompressible fluids, and no capillary forces, the upscaled relative permeabilities and capillary pressure are linear functions of $S$. If we, for a moment, disregard residual and solubility trapping, there are three main factors that qualitatively change the upscaled relative permeabilities and capillary pressures (see Table 1): (i) vertical heterogeneity in permeability and porosity, (ii) capillary pressure at the fine scale, and (iii) compressibility. Compared with traditional black-oil models, the new qualitative feature introduced is that relative permeabilities and capillary pressure may become pressure dependent for certain combination of these three effects, as summarized in Table 1. Adding an extra functional dependence will increase the computational cost of the property evaluation, while introducing a nonlinearity will generally cause a stronger coupling between the flow and transport equations in the VE model. Small-scale undulations in the caprock will also impact effective relative permeabilities, as explained in [8].

A formulation of the VE model in terms of saturations, compatible with the standard black-oil framework, would only require minimal changes to conventional reservoir simulators and could therefore be considered the most practical choice. From a numerical point-of-view, this gives accumulation terms $(H \Phi S)$ that are simple to evaluate: $S$ is the primary unknown whereas $H$ and $\Phi$ are functions of the spatial variables and can be computed by preprocessing the geological model. On the other hand, to evaluate the effective mobilities by integrating along the vertical columns of the underlying 3D grid, we need the vertical fluid distribution which can be computed from Eq. 5 once $p_{c}$ is known. To compute the capillary pressure from Eq. 4, we must first compute $h$ by inverting $S_{n}(h)$, which requires solving at least one nonlinear equation. This problem is avoided entirely if the model is formulated in terms of plume height because computation of capillary pressure, vertical fluid distribution, and effective mobilities can then be based on function evaluations only. On the other hand, the accumulation term now requires evaluation of an integral along the vertical column to determine $S_{n}(h)$.

A simple solution to speed up the computation of nonlinear functions is to use precomputed tables. To treat the general case rigorously, such tables will be four-dimensional to reflect the dependency on $p, H, S$, and $S_{\max }$ for homogeneous cases. Here, $S_{\max }$ represents the historical maximum value of $S$, and its role is further discussed in Section 5. For heterogeneous media, a separate three-dimensional table for each column would be needed. In other words, a fully rigorous treatment by precomputed tables would require more multidimensional tables than what is practical and efficient for large-scale simulations and require function evaluation at many points that will not be seen in the simulation. To obtain a practical method, we have therefore implemented different approximations in MRST-CO2lab to speed up the evaluation of effective properties and generally make the $S$ formulation of VE models more computationally tractable. Table 2 summarizes some cases where simplified models can be obtained. We have found the sharp-interface model (first row) and the capillary fringe approximation (third
Table 2 Table of simplified models for relative permeability
Compressible Rock param. vert. Capillary Aquifer height Representation

\begin{tabular}{lllll}
\hline yes & constant & no & & analytic, closed form \\
no & constant & yes & $H \equiv$ const & three simple tables of saturation \\
yes & constant & yes & $h<H$ & three 1D tables: $\widetilde{v}(\Delta p), \widetilde{k}_{r w}(\Delta p), \widetilde{k}_{r n}(\Delta p)$ \\
yes & varying & no & & calculate $p_{c}, k_{r w}, k_{r n}$ by sums for each column \\
\hline
\end{tabular}


row) to be particularly important. The use of one of these simplified models will be sufficient in many cases, especially given the general lack of detailed knowledge about large-scale aquifers.

\subsection{Vertically homogeneous porosity and permeability}

If we assume a vertically homogeneous case, it is possible to transform all the integrals in Eq. 6 so that the integrands are independent of the density difference. To this end, we define a new integration variable $p=g \Delta \rho(z-h)$, set $\Delta p=$ $g h \Delta \rho=P_{c}-p_{c, i}$, and introduce the function $\hat{s}_{\mathrm{n}}(\cdot)=(1-$ $\left.\hat{p}_{c}^{-1}\right)(\cdot)$. Then, using (5) and assuming that $h<H$, we see that the integrals in Eq. 6 can be written as,

$$
\begin{aligned}
S_{\mathrm{n}}(\Delta p) & =\frac{1}{g H \Delta \rho} \int_{0}^{\Delta p} \hat{s}_{\mathrm{n}}\left(p_{c, i}+p\right) \mathrm{dp} \\
\Lambda_{\mathrm{n}}(\Delta p) & =\frac{1}{g H \Delta \rho} \int_{0}^{\Delta p} \lambda_{\mathrm{n}}\left(\hat{s}_{\mathrm{n}}\left(p_{c, i}+p\right)\right) \mathrm{dp} \\
\Lambda_{w}(\Delta p, h) & =\frac{H-h}{H} \lambda_{w}(1)+\frac{1}{g H \Delta \rho} \int_{p_{c, m}}^{p_{c, i}+\Delta p} \lambda_{w}\left(\hat{p}_{c}^{-1}(p)\right) \mathrm{dp} .
\end{aligned}
$$

The integrals in Eq. 7 are independent of the aquifer height and the density difference (and consequently the global pressure) and can be efficiently implemented using three one-dimensional arrays tabulating them as functions of the pressure difference across the depth of the plume. Notice that as long as $h<H$, Eq. 7 is only different from Eq. 6 if the density is not constant within the column. By using the inverse of the $S_{\mathrm{n}}$ table, the tables for the effective mobilities can alternatively be precomputed as functions of $S$. When used as an approximation, Eq. 7 will be referred to as $P$-scaled table. In the rare case that $h>H$ (i.e., when saturation at $z=H$ is positive), the limits of the integrals in Eq. 7 are incorrect. To obtain values that are always correct, we need to subtract integrals over $[0, g \Delta \rho \max (h-H, 0)]$, which also can be expressed in terms of the original tables and replace $h$ by $\min (h, H)$ in the first term of $\Lambda_{w}$. Unfortunately, this system cannot be calculated by only inverting the table, but will require solving a nonlinear system. An approximate, but more computationally efficient approach is to use a linear approximation in this zone. In the case that the fine-scale model has multiple rock types that each has different relative permeability and capillary pressure curves, a unique set of tables must be developed for each rock type.
The expression above does not take into account the fact that the capillary pressure depends on permeability. To introduce this effect, the fine-scale capillary pressure can be approximated in terms of a Leverett J-function, scaled by the permeability,

$\hat{p}_{c}\left(s_{w}\right)=\sigma \cos (\theta) \sqrt{\frac{\phi}{|\mathbf{K}|}} J\left(s_{w}\right)=\hat{C}_{p}^{-1} J\left(s_{w}\right)$,

where $\sigma$ is surface tension and $\theta$ the contact angle. The inverse mapping from capillary pressure values to saturation can now be approximated by $\hat{p}_{c}^{-1}(p) \approx J^{-1}\left(\hat{C}_{p} p\right)$. Introducing the variable change from $p$ to $y=\hat{C}_{p} p$ in the integral in Eq. 7, we can calculate all quantities for all columns using only three tables, even if permeabilities differ between grid columns. These tables will be tabulated in values of $\hat{C}_{p} p$. The resulting approximation will be referred to as $P$-K-scaled table.

\subsection{Linear capillary fringe}

To investigate the effect of a capillary fringe, a simple linear model for the fringe is instructive. A more complicated physical based model can be found in [32]. If we define the height of the fringe as $h_{c}=\left(p_{c, m}-p_{c, i}\right) / g \Delta \rho$, there will be four different types of vertical saturation distributions as illustrated in Fig, 5.

The fine-scale relative permeabilities are assumed to follow a Corey-type model, i.e., $\hat{k}_{r, \mathrm{n}}\left(s_{e}\right)=k_{\mathrm{n}}^{e}\left(1-s_{e}\right)^{n_{\mathrm{n}}}$ and $\hat{k}_{r, w}\left(s_{e}\right)=k_{w}^{e} s_{e}^{n_{w}}$, where $s_{e}=\left(s_{w}-s_{w, r}\right) /\left(1-s_{w, r}\right)$. Let us first consider the case in Fig. 5a. Assuming that $\phi$ and $\mathbf{k}$ are constant in the vertical direction, we derive an expression for the upscaled saturation as follows:

$$
\begin{aligned}
S_{n}(h) & =\frac{1}{H} \int_{0}^{H} s_{n}(z) \mathrm{dz}=\frac{1}{H} \int_{0}^{h}\left(\frac{h}{h_{c}}\left(1-s_{w, r}\right)\right)\left(1-\frac{z}{h}\right) \mathrm{dz} \\
& =\frac{1-s_{w, r}}{H} \int_{0}^{h} \frac{h-z}{h_{c}} \mathrm{dz}=\frac{\left(1-s_{w, r}\right)}{H} \frac{1}{2}\left(\frac{h}{h_{c}}\right)^{2}
\end{aligned}
$$

The derivations for the other parameters and cases are similar. To summarize all four cases, we first define the function

$$
f(h, n)= \begin{cases}\frac{h_{c}}{n+1}\left(\frac{h}{h_{c}}\right)^{n+1}, & \text { if } h<\min \left(h_{c}, H\right), \\ \frac{h_{c}}{n+1}\left[\left(\frac{h}{h_{c}}\right)^{n+1}-\left(\frac{h-H}{h_{c}}\right)^{n+1}\right], & \text { if } H<h<h_{c}, \\ \frac{h_{c}}{n+1}+\left(h-h_{c}\right), & \text { if } h_{c}<h<H, \\ \frac{h_{c}}{n+1}\left(1-\left(\frac{H-h}{h_{c}}\right)^{n+1}\right)+h-h_{c}, & \text { if } \max \left(h_{c}, H\right)<h,\end{cases}
$$


Fig. 5 The four types of vertical saturation distribution arising for a model with a linear capillary fringe. Here, $H$ is the aquifer height, $h$ is the height of the plume, and $h_{c}$ is the height of a fully developed capillary fringe

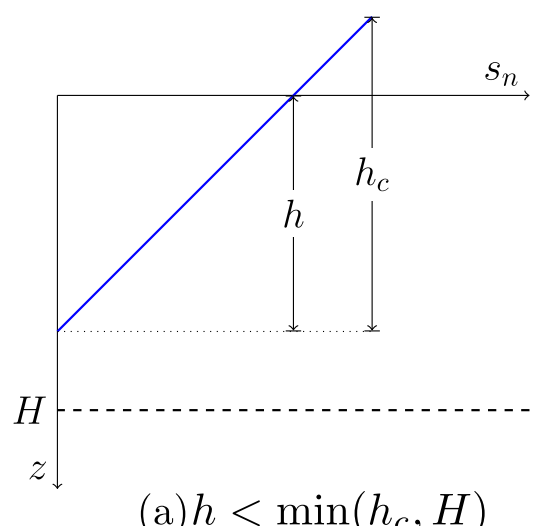

(a) $h<\min \left(h_{c}, H\right)$

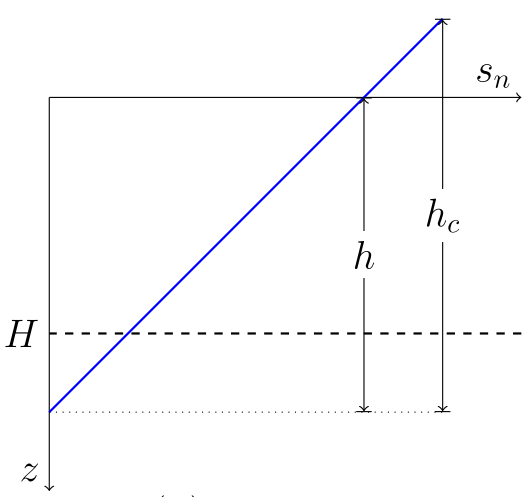

(b) $H<h<h_{c}$

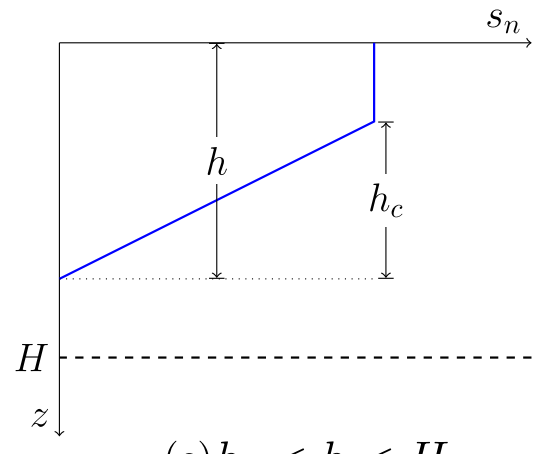

(c) $h_{c}<h<H$

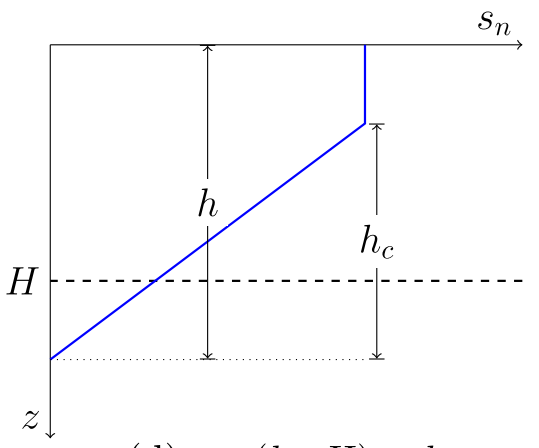

(d) $\max \left(h_{c}, H\right)<h$ and then write the upscaled quantities as

$$
\begin{aligned}
S_{\mathrm{n}}(h) & =\frac{\left(1-s_{w, r}\right)}{H} f(h, 1) \\
\Lambda_{\mathrm{n}}(h) & =\frac{\lambda_{\mathrm{n}}^{e}}{H} f\left(h, n_{\mathrm{n}}\right) \\
\Lambda_{w}(h) & =\frac{\lambda_{w}^{e}}{H} f\left(H-h+h_{c}, n_{w}\right) .
\end{aligned}
$$

\subsection{Comparison of models}

If we summarize our discussions so far, we have introduced five different ways of reconstructing the vertical fluid distribution and computing effective properties for use in the averaged flow equations:

- the sharp-interface model presented in [8];

- linear capillary fringe (9);

- P-scaled table (7) with effective properties tabulated against $\Delta p$

- P-K-scaled table: same as P-scaled but with capillary pressure given by a Leverett-J function scaled by $\mathbf{K}$;

- S table: the exact relationships (5) and (6).
To compare these models, we will consider a simple example.

Example 1 As our fine-scale model, we assume a homogeneous rock and use Corey type relative permeabilities $k_{r \alpha}=s_{\alpha}^{3}$ with end-point scaling so that $k_{r \mathrm{n}}\left(1-s_{w, r}\right)=$ 1 . The relationship between saturation and capillary pressure is assumed to be on the form $\hat{p}_{c}(s) \propto s^{-1 / 2}$ (i.e., a Brooks-Corey type curve with $\alpha=1 / 2$ ), which gives us the following inverse functions

$$
\begin{aligned}
& \hat{p}_{c}^{-1}(p)=\max \left[\left(\frac{1}{1+\hat{C}_{p} p}\right)^{2}, s_{w, r}\right], \\
& \hat{J}_{c}^{-1}(\tilde{p})=\max \left[\left(\frac{1}{1+\tilde{p}}\right)^{2}, s_{w, r}\right] .
\end{aligned}
$$

Moreover, we assume $\sigma \cos (\theta)=30 \mathrm{mPa}$, porosity 0.1 , and permeability $100 \mathrm{mD}$. The scaling parameter $\hat{C}_{p}$ is set to $(0.4 \mathrm{~g} \Delta \rho H)^{-1}$, and the height of the capillary fringe is set to $h_{c}=0.3 H$.

Figure 6 shows reconstructed saturation distribution, relative permeability for $\mathrm{CO}_{2}$, and coarse-scale capillary pressure for the case with $h=15 \mathrm{~m}, H=25 \mathrm{~m}$, and 
$s_{w, r}=0$. For the sharp-interface model, the saturation is zero below $h=15$ and one above, giving a linear relative permeability. For the other models, we notice the nonlinearity of the upscaled relative permeabilities and capillary pressure for small saturations. For the linear capillary case, the saturation change across the linear fringe is equal $h_{c} / 2 H$, and hence, the upscaled functions will only be nonlinear for $s<\left(1-s_{w, r}\right)\left(h_{c} / 2 H\right)$, which equals 0.15 in this example. For the other models, the transition to a linear regime is more gradually and happens for plume heights $h<C /(\Delta \rho g H) \approx 0.4$, or equivalently in the saturation range for which upscaled capillary pressure is comparable in value to the fine-scale capillary pressure. This transition regime can be seen on the lower plot in Fig. 6 for the region $s<0.2$.

The discrepancies between the relative permeability and the capillary curves for the P-K-scaled model, on one hand, and the P-scaled and the exact curves, on the other, are small for thin plumes. Moreover, we see that the P-K scaled table has a bit smaller capillary fringe than the P-scaled table and the exact case, because of smaller capillary pressure. The advantage of using $\mathrm{P}$-scaled tables is that the curves for different densities and aquifer thicknesses can be calculated using only a single 1D table. The additional advantage of the P-K-scaled table is that varying rock properties can be handled without using additional tables (assuming the relationship (8)). The discrepancy in relative permeabilities between P-scaled table and the exact model can be explained by the fact that we can only compute table values for $h \leq H$, which corresponds approximately to $s \leq$ 0.7. Therefore, a linear interpolation is used in the region $s>0.7$, which corresponds to the case in which the plume height would extend beyond the depth of the aquifer.

\section{Hysteresis effects}

Residual trapping is a first-order effect for $\mathrm{CO}_{2}$ migration that will generally lead to irreversibility, also known as hysteresis [40], in the vertical-equilibrium models, see, e.g., [27, 32]. In the models discussed herein, the residually trapped volume is determined by the difference between $S_{\max }$ and $S_{\mathrm{n}}$, and this difference is inherently linked to hysteretic effects. In sharp-interface models, residual trapping is simple to handle because the local reconstruction of fluid distribution in the vertical columns of the underlying 3D model only has three valid saturation regimes: maximum $\mathrm{CO}_{2}$ concentration, residual $\mathrm{CO}_{2}$ concentration, or no $\mathrm{CO}_{2}$. Hence, it is sufficient to track the height $h$ of the $\mathrm{CO}_{2}$ plume and the height $h_{\max }$ at which $\mathrm{CO}_{2}$ has existed throughout the simulation history to define the vertical saturation distribution:

$s_{\mathrm{n}}(z)= \begin{cases}1-s_{w, r}, & z \leq h \\ s_{n, r}, & h \leq z \leq h_{\max } \\ 0, & h_{\max } \leq z\end{cases}$

Each of these zones has unique mobilities that are independent of the history if the hysteresis model used in the fine-scale 3D description only depends on $s$ and the maximum value $s_{\max }$ seen over the saturation history. If we let $\lambda_{\alpha}^{e}$ denote the end-point of mobility curve $\lambda_{\alpha}$ and define
Fig. 6 The figure shows reconstructed saturation distributions for $h=15 \mathrm{~m}$ (left), upscaled relative permeability for $\mathrm{CO}_{2}$ (right), and upscaled capillary pressure with the $y$-axis scaled by $g \Delta \rho H$ (bottom). The height of the given column is $H=25 \mathrm{~m}$. (From: plotVEReconstruction.m)
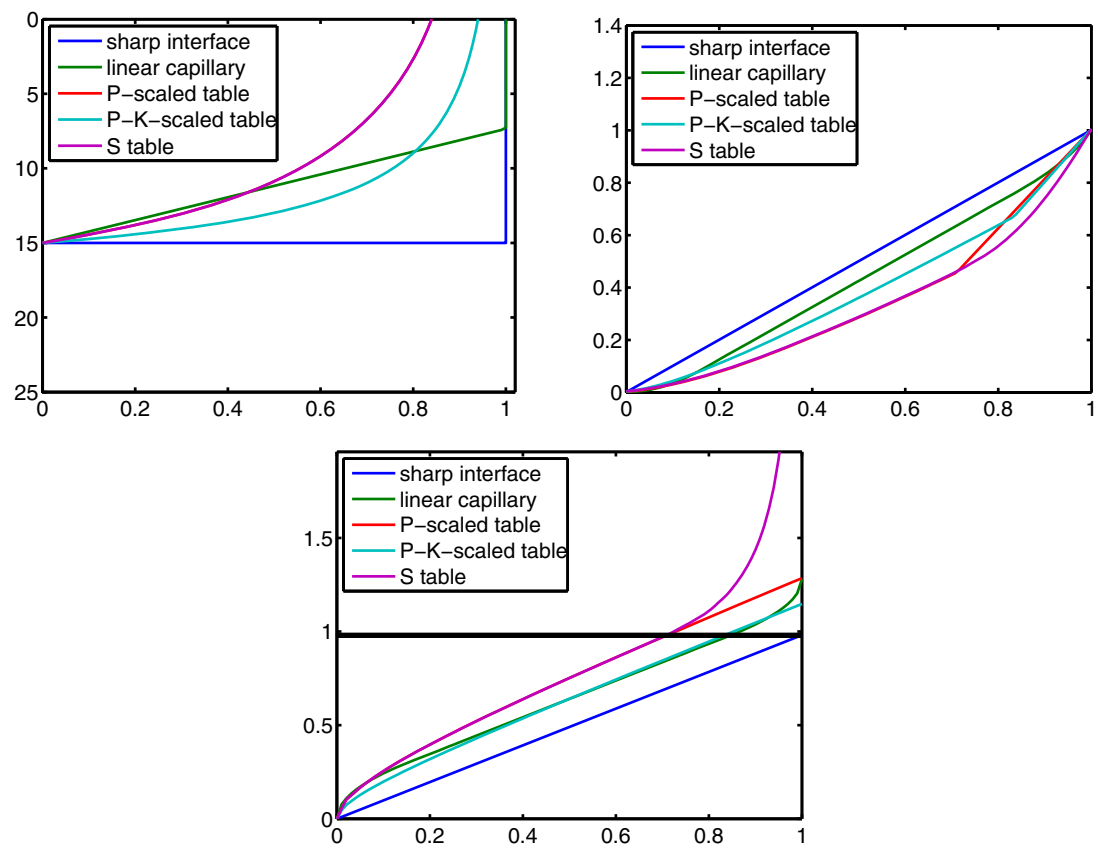
$\Phi(z)=\frac{1}{H} \int_{0}^{z} \phi\left(z^{\prime}\right) d z^{\prime}$ and likewise for $\mathbf{K}(z)$, the upscaled properties in Eq. 6 can be seen to follow a traditional hysteresis model that has a particular simple form:

$$
\begin{aligned}
S_{\mathrm{n}} & =\left[\Phi(h)\left(1-s_{w, r}\right)+\left(\Phi\left(h_{\max }\right)-\Phi(h)\right) s_{\mathrm{n}, r}\right] / \Phi(H) \\
S_{\max } & =\Phi\left(h_{\max }\right)\left(1-s_{w, r}\right) / \Phi(H), \\
\Lambda_{\mathrm{n}} \mathbf{K} & =\lambda_{\mathrm{n}}^{e} \mathbf{K}(h), \\
\Lambda_{w} \mathbf{K} & =\left[\left(\mathbf{K}(H)-\mathbf{K}\left(h_{\max }\right)\right) \lambda_{w}(1)+\left(\mathbf{K}\left(h_{\max }\right)-\mathbf{K}(h)\right) \lambda_{w, r}\right], \\
P_{c} & =g h \Delta \rho .
\end{aligned}
$$

While the hysteretic behavior of $S_{\mathrm{n}}$ and $\Lambda_{w}$ is obvious from the expressions above and will appear explicitly in a pure $h$-formulation, the hysteretic behavior of $\Lambda_{\mathrm{n}}$ and $P_{c}$ follows implicitly from the fact that $h$ now also depends on $S_{\max }$. In particular, whereas $P_{c}$ is a linear function of $h$, in the $S$ formulation, it is a much more complex function $\hat{P}_{c}\left(S_{n}, S_{\max }, P\right)$.

The problem gets more complicated when the underlying model has a capillary fringe, since the shape of the fringe then generally will depend on previous profiles. If the fine-scale capillary pressure follows a hysteretic curve $\hat{p}_{c}\left(s_{w}, s_{w, \max }\right)$, where $s_{w, \max }=1-s_{\max }$, the expressions in Eq. 6 for the averaged quantities are still valid when $s_{w}=s_{w, \max }$. In the general case, the fine-scale saturation is reconstructed as follows:

$$
\begin{aligned}
p_{c, \max }\left(s_{w}\right) & =\hat{p}_{c}\left(s_{w}, s_{w}\right) \\
s_{\max }(z) & =1-p_{c, \text { max }}^{-1}\left(p_{c}\left(z ; h_{\max }\right)\right) \\
S_{\max } & =\frac{1}{H} \int_{0}^{h_{\max } 1-p_{c, \text { max }}^{-1}(z) \mathrm{dz}} \\
s_{\mathrm{n}}(z) & = \begin{cases}\hat{s}_{\mathrm{n}}\left(p_{c}(z ; h), s_{w, \max }(z)\right), & z<h, \\
s_{\mathrm{n}}^{e}\left(s_{\max }(z)\right), & h<z<h_{\max }, \\
0, & h_{\max }<z,\end{cases}
\end{aligned}
$$

where $\hat{s}_{\mathrm{n}}(\cdot, \cdot)=\left(1-\hat{p}_{c}^{-1}\right)(\cdot, \cdot)$ and the lower endpoint $s_{\mathrm{n}}^{e}\left(s_{\max }\right)$ on the relative permeability curve emanating from $s_{\max }$ is an analogy to the residual saturation seen in the sharp-interface models. In the case of a general function $\hat{p}_{c}\left(s_{w}, s_{w, \max }\right)$, the reconstruction in Eq. 13 must be computed on the fly by solving a set of nonlinear problems.

The simpler case of a vertically homogeneous aquifer and a plume that never extends to the full height of the aquifer was investigated recently by Gasda et al. [31], who concluded that the main hysteretic effects can be handled by simplified trapping models and that the specific form of the model is less important than capturing the reduced mobility and increased coarse-scale 'capillary pressure' of $\mathrm{CO}_{2}$ for small plume heights. The effects of different finescale models are mainly captured if the simple hysteretic model accounts for the nonlinearity of the relative permeability caused by the capillary fringe and the correct amount of effective residually trapped $\mathrm{CO}_{2}$. In the rest of the section, we will discuss simplified hysteresis models that enable efficient reconstructions based on tabulated values, much in the same way as discussed in Section 4.

\subsection{Endpoint-scaling model}

We start by assuming that all scanning curves in the finescale relative permeability and capillary pressure functions have the same functional form, which implies that the scanning curves are 'reversible' in the sense that the imbibition curve also models a subsequent drainage process. Moreover, we assume that the residual endpoint of each scanning curve for the relative permeability is defined as

$s_{\mathrm{n}}^{e}\left(s_{\max }\right)=\frac{s_{\mathrm{n}, r}}{1-s_{w, r}} s_{\max }=C s_{\max }$,

e.g., as illustrated in Fig. 7. If $\hat{k}_{r, \mathrm{n}}$ denotes the primary relative permeability curve for $s \in[0,1]$, then the scanning curve for $s \in\left[s_{\mathrm{n}}^{e}\left(s_{\max }\right), s_{\max }\right]$ is defined as $k_{r, \mathrm{n}}\left(s, s_{\max }\right)=$ $\hat{k}_{r, \mathrm{n}}\left(\hat{s}_{\text {eff }}(s)\right)$, where the rescaled saturation variable used to define the scanning curve reads

$\hat{s}_{\text {eff }}(s)=s_{\max } \frac{s-s_{\mathrm{n}}^{e}}{s_{\max }-s_{\mathrm{n}}^{e}}=s-\frac{C}{1-C}\left(s_{\max }-s\right)$
Fig. 7 Left: Illustration of a simple fine-scale relative permeability function with hysteresis effects, in which the scanning curve between $s_{\mathrm{n}}^{e}\left(s_{\max }\right)$ and $s_{\max }$ has the same functional form as the primary curve between 0 and 1 . Right: Reconstruction of vertical saturation distribution
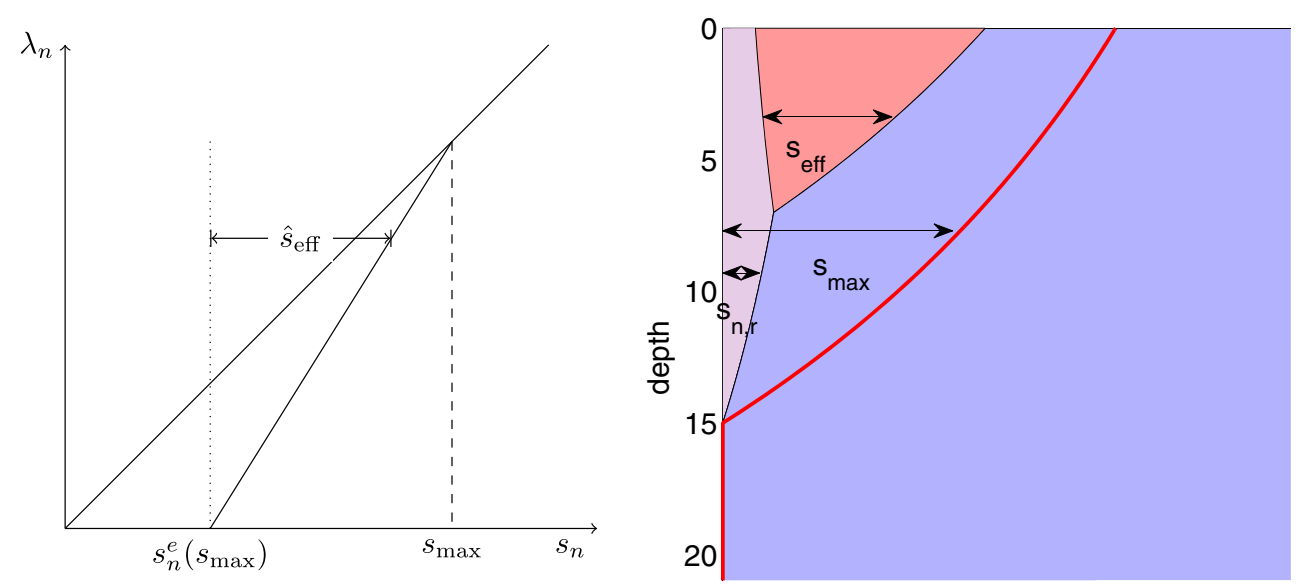
for the case when $\hat{k}_{r}$ is linear as in Fig. 7. In the general case, we will use this as an approximation so that if we know $s(z)=1-s_{w}(z)$ from Eq. 5 and $s_{\max }(z)$ from Eq. 13, we can compute $s_{\text {eff }}(z)$ as follows

$s_{\mathrm{eff}}(z)=s(z)-\frac{C}{1-C}\left(s_{\max }(z)-s(z)\right)$,

which translates to the following expression for the averaged saturations,

$S_{\text {eff }}\left(h, h_{\max }\right)=S\left(h, h_{\max }\right)-\frac{C}{1-C}\left(S_{\max }\left(h_{\max }\right)-S\left(h, h_{\max }\right)\right)$.

From the assumptions of $\hat{p}_{c}\left(s_{\text {eff }}\right)$, we can now find $h$, $h_{\max }$ and consequently $s_{\mathrm{eff}}(z)$ and $s_{\max }(z)$. Then, we have all we need to reconstruct the vertical saturation distribution and compute the upscaled mobilities. In particular, the saturation distribution reads,

$s_{\mathrm{n}}(z)= \begin{cases}s(z), & z \leq h, \\ C s_{\max }(z), & h \leq z \leq h_{\max } \\ 0, & h_{\max } \leq z\end{cases}$

For $z<h$, the saturation that is already residually trapped is given by $s(z)-s_{\text {eff. Including hysteretic effects does not }}$ change the capillary-fringe model for $h_{\max }=h$, and the new model reduces to the sharp-interface model in the case of a constant capillary pressure function. The model also has the properties that the residually trapped $\mathrm{CO}_{2}$ in a column will be $C S_{\max }=s_{\mathrm{n}, e} S_{\max } /\left(1-s_{w, e}\right)$, which will be the case in any model in which the residual endpoint of the relative permeability is $s_{\mathrm{n}, e} s_{\max } /\left(1-s_{w, e}\right)$.

In the $S$-formulation, $h$ and $h_{\max }$ are not known and must be computed from $S, S_{\max }$, and $P$. To compute $h$, we start by observing that since all scanning curves have the same functional form, the fine-scale capillary pressure will be a function of $s_{\text {eff }}$. Replacing $s_{n}$ by $s_{\text {eff }}$, we can either use the relationship between $\hat{p}_{c}^{-1}$ and $S_{n}$ given by Eq. 5 and 6 to form a nonlinear equation for $h$, or alternatively use a precomputed tabular that implements one of the (approximate) relationships discussed in Section 4. For instance, if we choose to use Eq. 7, $h$ and $h_{\max }$ can be computed from the global pressure $P$ and the precomputed, P-scaled table $\hat{S}_{n}(\Delta p)=g H \Delta \rho S_{n}(\Delta p)$ as follows

$$
\begin{aligned}
h & =\frac{1}{g \Delta \rho(P)} \hat{S}_{n}^{-1}\left(g H \Delta \rho(P) \cdot S_{\mathrm{eff}}\right), \\
h_{\max } & =\frac{1}{g \Delta \rho(P)} \hat{S}_{n}^{-1}\left(g H \Delta \rho(P) \cdot S_{\max }\right) .
\end{aligned}
$$

We can now insert $h$ in Eq. 4 to compute the capillary pressure $p_{c}(z)$, from which we get $s_{\mathrm{eff}}(z)=\hat{p}_{c}^{-1}\left(p_{c}(z)\right)$, and similarly we get $s_{\max }(z)$ by inserting $h_{\max }$ instead of $h$. Inserting $s_{\text {eff }}(z)$ and $s_{\max }(z)$ into Eq. 16 and solving for $s(z)$, we finally can compute the vertical saturation distribution from Eq. 18.

\subsection{Sharp-interface type model}

Another model can be obtained if we assume that the capillary fringe has exactly the same shape as for the case without hysteresis. For this to be possible, the effective saturation used to evaluate the fine-scale relative-permeability curves has to have a drop from the maximum residually trapped $\mathrm{CO}_{2}$ saturation to zero at $h$. In other words,

$$
\begin{aligned}
& s(z)= \begin{cases}\hat{s}_{n}\left(p_{c}(z)\right), & z<h \\
s_{n, r}, & h<z<h_{\max } \\
0, & h_{\max } \leq z,\end{cases} \\
& k_{r, \mathrm{n}}(z)= \begin{cases}\hat{k}_{r, \mathrm{n}}(s(z)), & z<h, \\
0, & h<z .\end{cases}
\end{aligned}
$$

Likewise, the only way one can have a region of constant residual saturation between $h$ and $h_{\max }$ is if the capillary function $\hat{p}_{c}$ is constant for $s_{n} \in[0, \tilde{s}]$, where $\tilde{s} \geq s_{n, r}$. (In a sharp-interface model, $\tilde{s}=1$ by definition.) The capillary function is therefore distinctly different from the one used for the model in Section 5.2.

In the new model, the averaged saturation is expressed as

$S_{n}\left(h_{\max }, h\right)=S_{\mathrm{eff}}(h)+\frac{h_{\mathrm{max}}-h}{H} s_{\mathrm{n}, r}$

If we further assume that the relative permeabilities only depend on the effective residual saturation, and not on the maximum saturation, for values with $S>s_{\mathrm{n}}^{e}$, the original fine-scale curves can be used to evaluate the averaged properties.

Because $h$ and $h_{\max }$ are not known in the $S$-formulation, they must be computed from $S, S_{\max }$, and $P$. To this end, one can,for instance, use a P-scaled tabular (7) to give the following equation

$S=S_{n}(g \Delta \rho(P) \cdot h)+\frac{h_{\max }-h}{H} s_{\mathrm{n}, r}$.

This expression involves $h_{\max }$, which can be calculated beforehand using the same expression with $h=h_{\max }$.

As far as we understand, a model very similar to the one outlined above has previously been used in [24] to investigate $\mathrm{CO}_{2}$ trapping mechanisms in the Johansen Formation from the Norwegian North Sea.

\subsection{Comparison of models}

To illustrate the how the hysteresis effects discussed above change the effective properties of the VE equations, we will consider two examples. In the first example, we revisit the setup from Example 1. 
Example 2 Figure 8 illustrates hysteretic effects using the end-point scaling model applied to the basic setup from Example 1 for a case in which $h=7 \mathrm{~m}$ and $h_{\max }=15 \mathrm{~m}$. Starting with the sharp-interface model without a capillary fringe, we see that the imbibition curves are linear but with a steeper inclination than the drainage curves. Similarly, in the other models, the primary drainage curves have been shifted and scaled to describe the imbibition and a possible subsequent drainage process. We notice that also in the hysteretic case, the effective curves are mostly linear except for small plume heights.

In the third example, we will illustrate how different choices for the underlying fine-scale capillary function can change the reconstructed saturation distribution and the effective parameters in the coarse-scale model. Specifically, we will construct a special capillary function that will enable us the compare the endpoint-scaling model Section 5.1 and the sharp-interface type model from Section 5.2.

Example 3 As explained in Section 5.2, the only way one can define a model that has a constant residual saturation between $h$ and $h_{\max }$ is to have a capillary function that is constant on an interval. Using the same setup as Eq. 10 in Example 1, and setting $p=p_{c}-p_{c, i}$ so that $p=0$ at $z=h$,

$s_{w}(p)= \begin{cases}\max \left[\left(\frac{C}{C+p}\right)^{2}-\tilde{s}, s_{w, r}\right], & p>0 \\ 1, & p<0\end{cases}$

This will give a capillary pressure curve

$p_{c}\left(s_{w}\right)= \begin{cases}\infty, & s_{w}<s_{w, r}, \\ C\left(s_{w}+\tilde{s}\right)^{-\frac{1}{2}}-1+p_{i, n}, & s_{w, r}<s_{w}<1-\tilde{s}, \\ p_{i, n}, & 1-\tilde{s}<s_{w} .\end{cases}$
We assume that $k_{r \mathrm{n}}(s)=s^{3}$ and $H=25 \mathrm{~m}$, as in the previous examples, and set $\tilde{s}=s_{r, n}=0.2, C=1.6 \mathrm{~g} \Delta \rho \mathrm{H}$, and $\Delta \rho=400 \mathrm{~kg} / \mathrm{m}^{3}$. Figure 9 shows how the two different capillary functions and the two hysteresis models effect the reconstruction of fine-scale saturation. The corresponding effective properties are shown in Fig. 10.

Here, we notice in particular that the relative permeability for the sharp-interface model has a very strong dependence on the residual saturation parameter compared with the equivalent endpoint-scaling model with capillary pressure function (23). This is because the latter has a hysteresis model based on scanning curves, while the sharp-interface type model has not. With the endpoint-scaling framework it is also possible to have effective relative permeabilities that increases slower than linearly from $\Lambda_{\mathrm{n}}=0$ as shown in Fig. 10. For the sharp-interface type model, on the other hand, $\Lambda_{\mathrm{n}}$ has a finite and positive derivative at $\Lambda_{\mathrm{n}}=0$.

As noted before, a formulation in terms of primary variables $h$ and $h_{\max }$ would allow general hysteresis models to be calculated explicitly on the fly. In principle, this can also be done from saturation variables as well, but then one has to solve nonlinear equations involving functions that are defined in terms of integrals. The main advantage of using the endpoint-scaling model is that with this model one can calculate all quantities in terms of $1 \mathrm{D}$ tables under the assumption of homogeneous columns, and that one can include K-scaling and pressure variations, provided that certain approximations are made if the plume extends to the bottom of the aquifer. We also notice that the effect of smallscale caprock undulations can be included in terms of an accretion layer model (see [8]) that uses $h_{\text {eff }}=h-h_{\text {trap }}$ to evaluate the effective relative permeability. The capillary pressure, however, is still computed using $h$.

At the end, we will use a simple example to illustrate the effect of dissolution on models including capillary fringe and small-scale geometrical trapping. For a discussion of the dissolution model itself, see [8] in which the dynamics
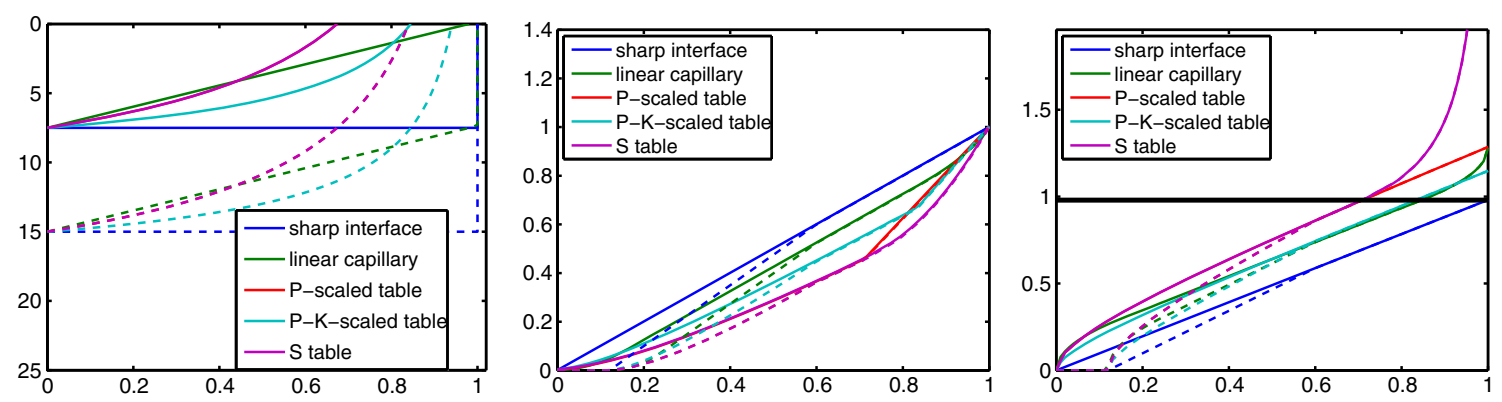

Fig. 8 The endpoint-scaling model applied to the basic setup from Example 1 as shown in Fig. 6. In the left plot, solid lines refer to $s_{e}(z)$ reconstructed from $h=7 \mathrm{~m}$, while dashed lines correspond to $s_{\max }(z)$. In the middle and right plot, solid lines are the drainage curves, whereas dashed lines are the imbibition curves for $S_{\max }=$ $\max (0.6, S)$. (From: plotVEReconstruction.m) 
Endpoint scaling $+(10)$
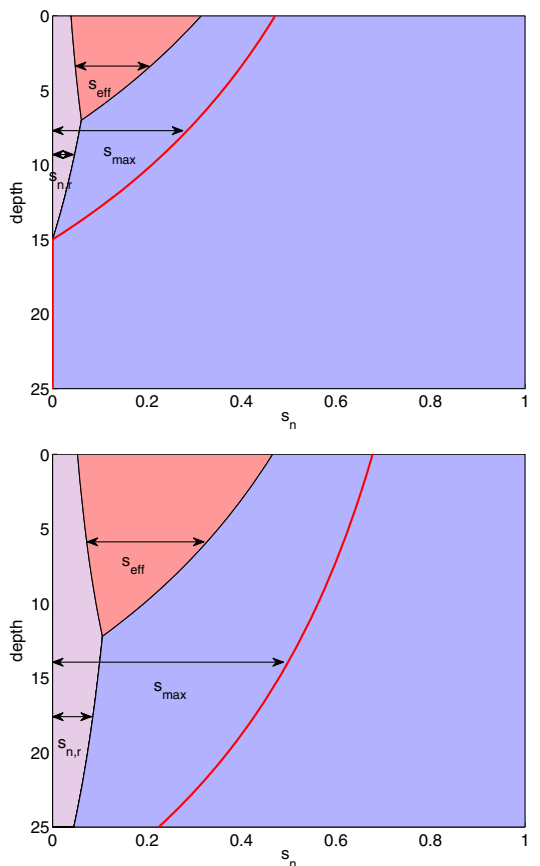

Endpoint scaling $+(23)$
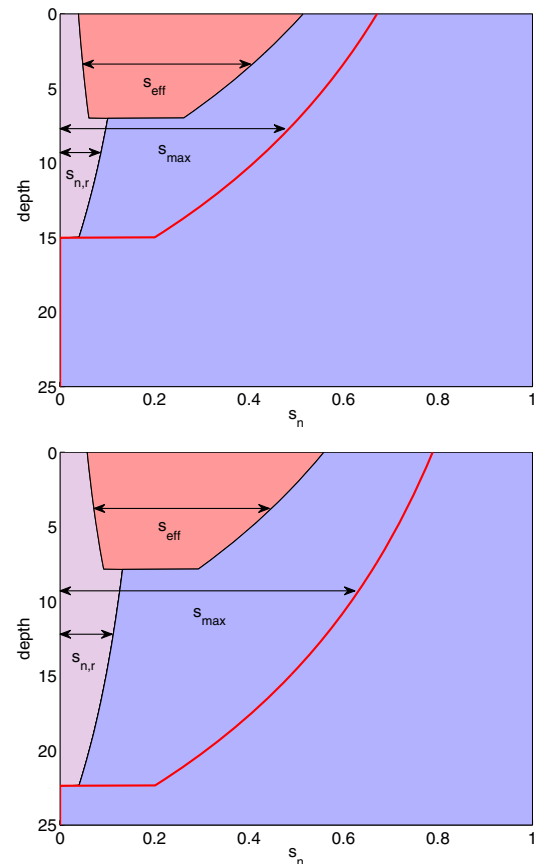

Sharp-interface type
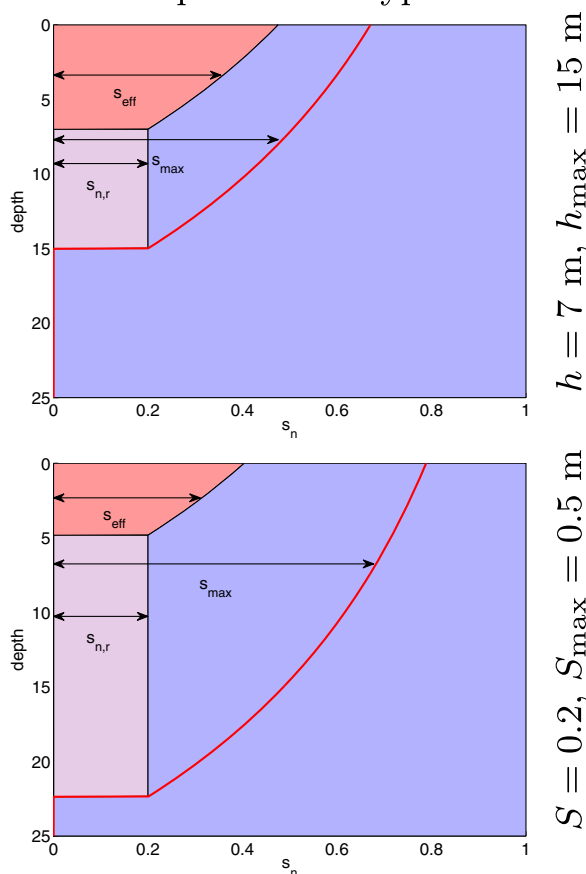

घ

10

II

Fig. 9 The figure illustrates how different assumptions for the underlying capillary-pressure curve change the vertical average model. The capillary-pressure and relative-permeability curves for the given models are shown in Fig. 10. (From: plotVEProfilesAndRelperms .m)

of $s_{\max }$ is discussed for the sharp-interface model. Again, we assume that the effect of dissolution is to first to reduce $s_{\max }$, which means that with instantaneous dissolution (i.e., a black-oil type model), $s_{\max }(t)$ will always be equal to the maximum saturation value observed during the previous imbibition process. With a finite dissolution rate, on the other hand, $s_{\max }(t)$ may be smaller than the maximum value

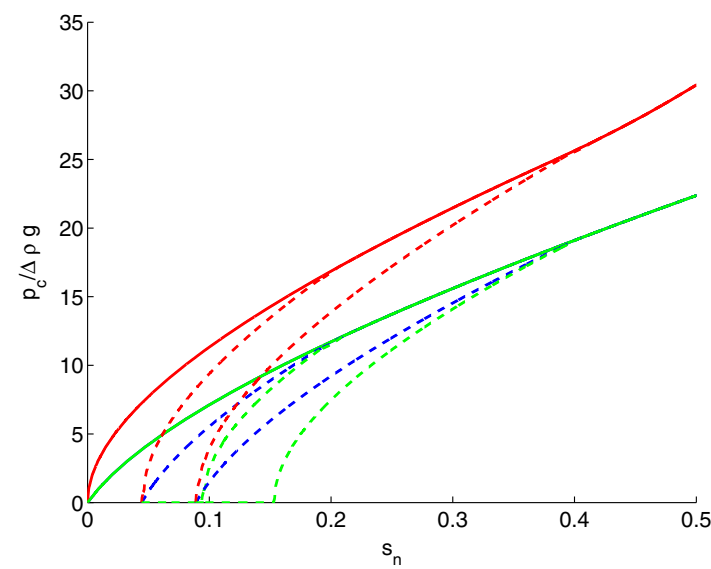

Fig. 10 Effective capillary pressure (left) and effective relative permeability (right) for the model three different models in Fig. 9. The solid lines are the primary imbibition curves and the dashed lines correspond to scanning curves for $S_{\max }$ equal 0.2 and 0.4 , seen during imbibition. To simplify our computations, we also make the approximation that the reconstruction from $s, s_{\max }$ can be done as for the case without dissolution. The main difference between a sharp-interface and a capillaryfringe model is that the depth of the plume will be larger for the latter, and hence more $\mathrm{CO}_{2}$ will be instantaneously dissolved.

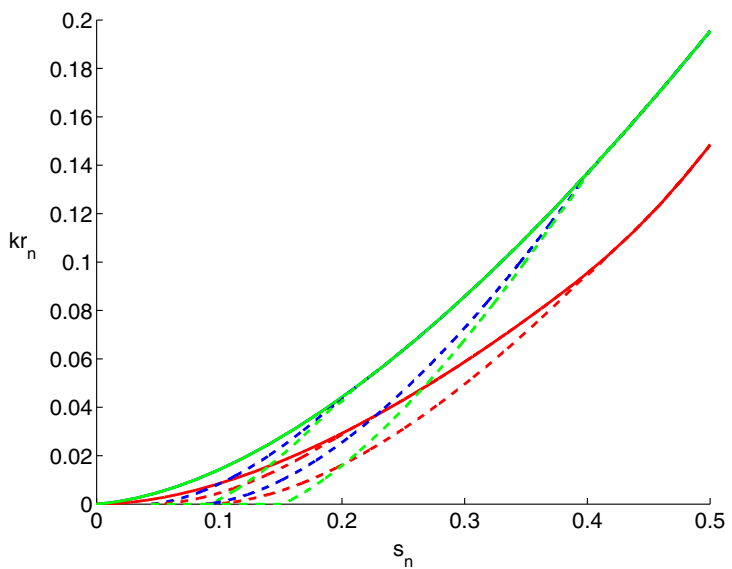

respectively. Green curves represent the sharp-interface type model, whereas blue and red curves represent the endpoint-scaling model with capillary pressure curves (23) and (10), respectively. (From: plotVEProfilesAndRelperms.m) 
330 years after injection
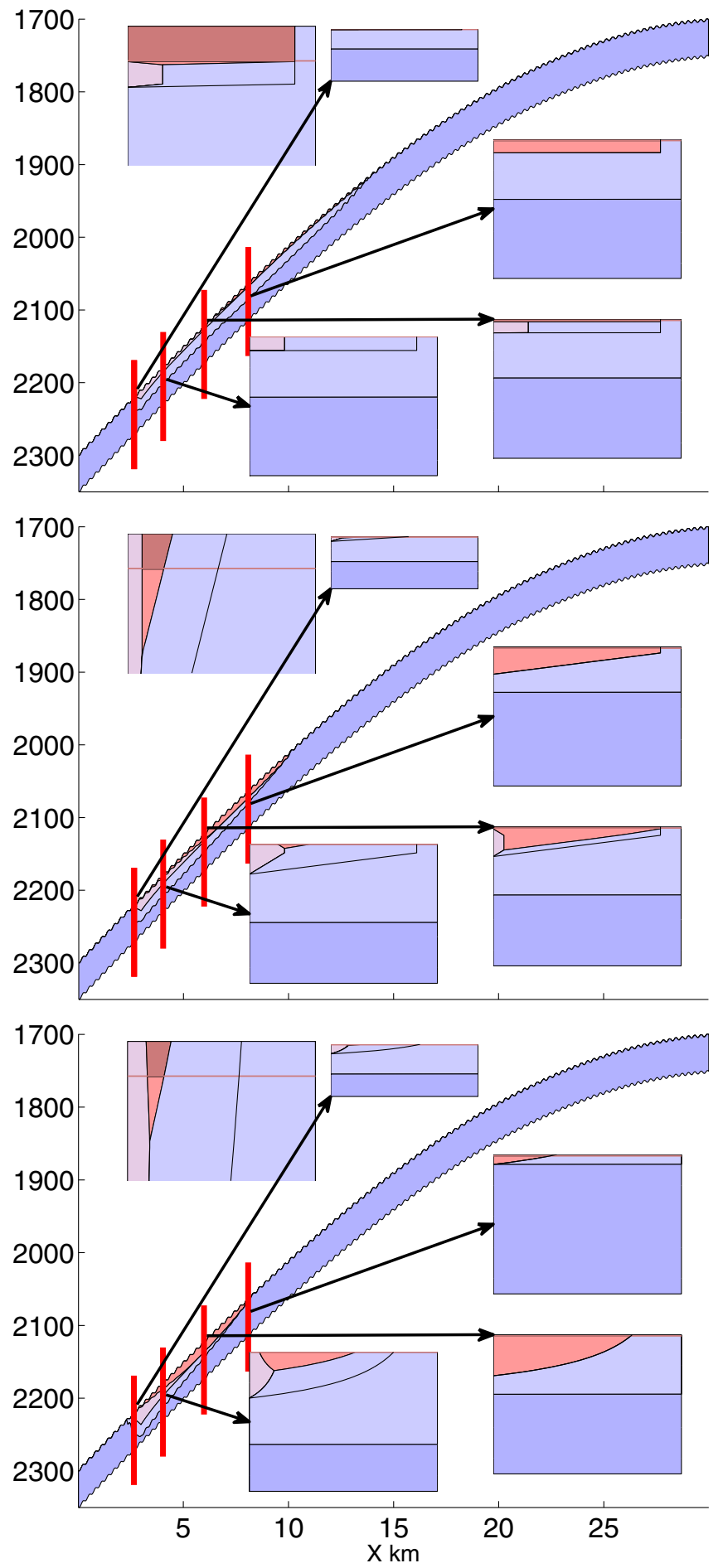

Fig. 11 The $\mathrm{CO}_{2}$ distribution in global coordinates 330 and 930 years after injection ceased in the simple 1D sloping aquifer. The main figure shows the full aquifer depth for scale, whereas the inlets is the reconstruction of the plume in four different cells of the model. The inlet
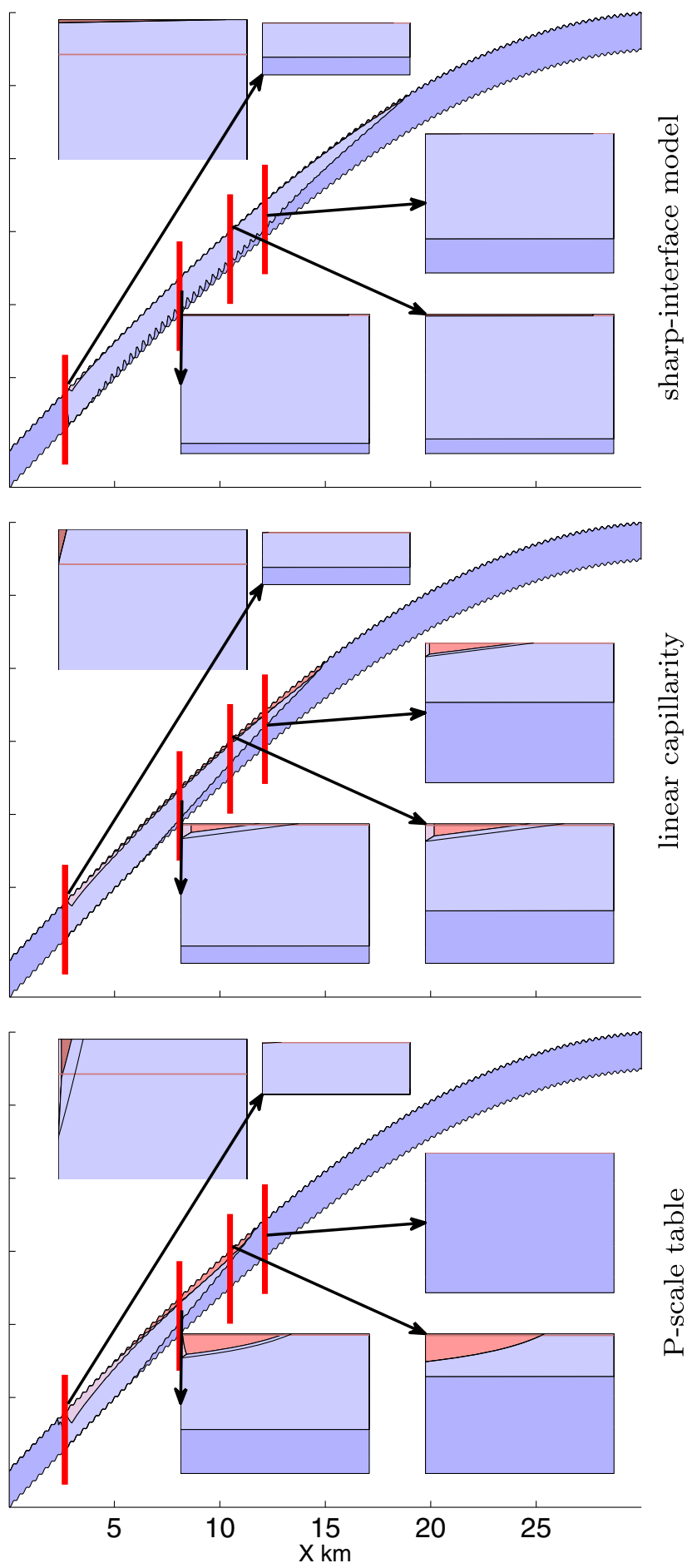

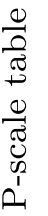

930 years after injection

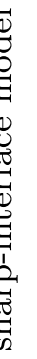

胥

dissolved brine trap height: -

in the top left corner of each model is a zoom of the first reconstruction for the depth of four times the trapping height, which was $0.4 \mathrm{~m}$. (From dissolutionExamplecomG2) 

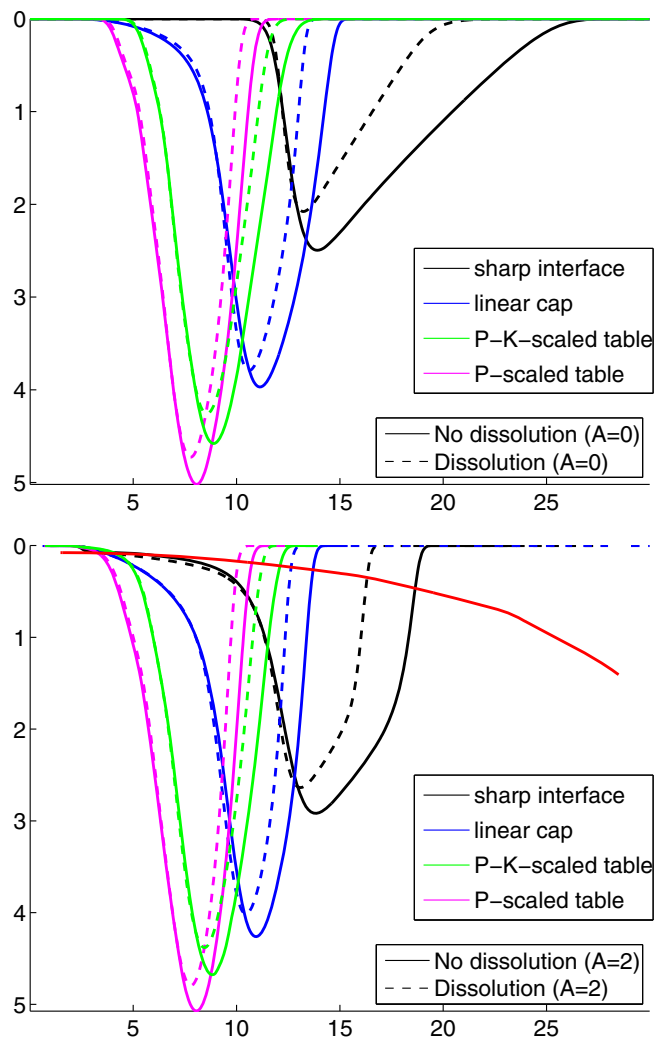

Fig. 12 The flowing $\mathrm{CO}_{2}$ computed by different fluid models for the simple 1D sloping aquifer. Colors signify different approximate methods for evaluating effective properties, dashed lines are models with dissolution, and solid lines are models without. In the upper plot, the top-surface is smooth $(A=0)$ and in the lower plot it has small-scale undulations $(A=2 \mathrm{~m})$. The red line shows the average height of the local traps (see [8, Fig. 10]). (From dissolutionExampleCOMG2)

Example 4 In this example, we consider a simple 1D model of a sealing caprock introduced in [8],

$z_{t}=D-L_{1} \sin \left(\frac{x}{L_{1}}\right) \tan \theta+A \sin \left(\frac{2 \pi x}{L_{2}}\right)$,

where $D$ is maximum depth, $\theta=0.03$ is the initial tilt angel, $L_{1}=20 \mathrm{~km}$ is the characteristic length of the antiform structure, and $L_{2}=0.3 \mathrm{~km}$ is the wave-length $L_{2} \mathrm{~m}$ of the small-scale structures, which we assume have an amplitude $A=0$ or $A=2 \mathrm{~m}$. The density of $\mathrm{CO}_{2}$ is assumed to follow a model taken from [41].

Figure 11 shows snapshots from three full simulations that include the coupled effect of dissolution and small-scale caprock undulations using three different models for finescale mobility and capillary pressure: sharp interface, linear capillary, and P-scaled table. We employ endpoint scaling for the hysteresis, which in the case of vanishing capillary fringe reduce to the sharp-interface hysteresis approach. In all simulations, we have used the dissolution rate taken from [8] for all models. In Fig. 12, we show the effective saturation, where we for completeness also have included results obtained using P-K-scaled tables. We see that the difference in the effective saturation for models with and without dissolution is much smaller at the trailing shock side. The reason for this is that the residual saturation has to be dissolved first, so the dynamics of the flowing part is relatively independent of the dissolution dynamics. However, dissolution clearly retards the imbibing front, and this effect is qualitatively the same for models with and without capillary fringe. Comparing the two subplots, we see that the capillary-fringe models are less retarded by small-scale trapping, and the actual amount of structurally trapped $\mathrm{CO}_{2}$ is also much smaller. We also see that the endpoint of the trailing rarefaction follows the local volumes of the sub-scale traps (shown as a red line in the figure) for the sharp-interface model, but falls below for the capillary-fringe models. This effect of the capillary fringe can be identified by comparing the amount of $\mathrm{CO}_{2}$ above the red line in the inlets of Fig. 11.

Example 5 To show the applicability of the models discussed herein, we compare the capillary fringe model with P-scaled table to a sharp-interface model in a large-scale, realistic setting. We use a simulation grid of the Utsira aquifer in the Norwegian North Sea to simulate the injection and migration of $\mathrm{CO}_{2}$ in a long-term perspective. We assume a simple setup in which $\mathrm{CO}_{2}$ is injected into the aquifer at two separate sites, indicated with black dots on the upper left plot in Fig. 13. Each site injects 150 megatons of $\mathrm{CO}_{2}$ over a period of 50 years, followed by a 1000 year migration period. During this period, some $\mathrm{CO}_{2}$ will be permanently trapped in residual form or within pockets (structural traps) of the caprock surface. The trapping state as a function of time is displayed in the two diagrams on the bottom row of Fig. 13. The positions of the corresponding free plumes are shown on the upper row. We observe that the sharp-interface model here results in a more irregularly-shaped plume as a result of increased gravity fingering, whereas the capillary fringe model spreads out more evenly. This can be explained by increased plume heights in this model (because of lower average saturation in each vertical column), which leads to a stronger upscaled capillary force that drives plume expansion in all directions.

The 2D grid used for the two VE simulations contains 23,393 cells, and each simulation ran within less than 5 min on a standard workstation PC. A full 3D simulation with a vertical resolution of 100 cells would thus have required 2.3 million cells, which in our experience leads to at least a factor ten larger runtimes. 

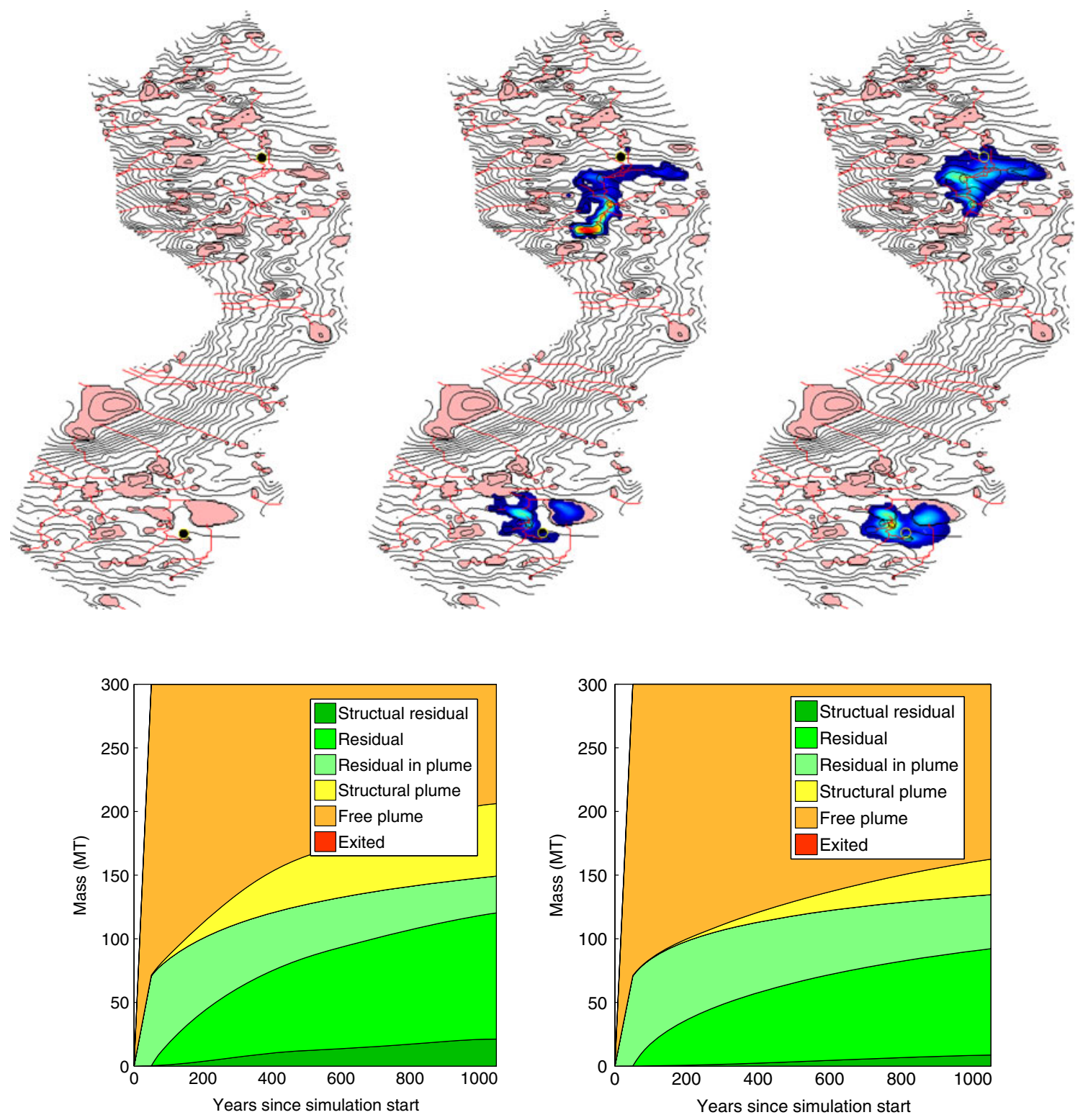

Fig. 13 Impact of different upscaled $\mathrm{CO}_{2}$ properties on a large-scale injection and migration scenario into the Utsira aquifer. Upper left: Topographical map of the Utsira caprock, with structural traps and spill paths indicated in red and injection points indicated with black dots. Upper middle and right: location of the free-flowing plume after 1000 years of migration using a sharp-interface (middle) and capillary fringe (right) model. Maximum plume thicknesses are $28.3 \mathrm{~m}$ (sharp

\section{Concluding remarks}

In the paper, we have presented a general, fully-implicit, and mass-conservative framework for VE models on unstructured grids that can be used to simulate the combined large-scale and long-term effects of structural, residual, and solubility trapping. The framework is implemented as part

interface) and $18.8 \mathrm{~m}$ (capillary fringe). Lower row: Corresponding breakdown of total injected $\mathrm{CO}_{2}$ into various states of trapping as a function of time. Left plot represents the simulation with a sharp interface, right plot with a capillary fringe. "Structural" trapping refers to the part of the plume contained within the structural traps of the caprock

of the free and open-source code MRST-CO2lab, which also includes the capabilities of constructing grid models from GIS data or the industry-standard corner-point format. As such, the software can be applied to perform highly resolved simulations of potential injection sites.

In the paper and in MRST-CO2lab, we mainly use model formulations that are as close as possible to standard 
black-oil formulations used by the petroleum industry. This is a pragmatic choice, motivated by the fact that if $\mathrm{VE}$ models are formulated in the black-oil framework, one can:

- easily include the VE models into existing commercial and academic simulators developed for this framework and potentially develop hybrid models that combine VE and 3D models in different parts of the domain,

- extend the VE models with the wide range of physical effects that are described in the literature using the black-oil framework, and

- utilize robust and reasonably efficient numerical methods developed over the past four decades. As discussed in more detail in [8], these methods are particularly suitable for accurately computing steady states that correspond to various kinds of trapping in the long-term transient migration of $\mathrm{CO}_{2}$.

Nevertheless, if one considers VE models from a pure computational point of view, it follows from the derivation and discussion above that the reconstruction of the fine-scale fluid distribution is more computationally efficient if the model is formulated in terms of $h, h_{\max }$. At present, we do not consider the computational gain to be worth the implementational cost and the added modeling complexity of using different primary unknowns for VE and 3D models. Likewise, an implementation of a general code in $h, h_{\max }$ with possible extensions to higher order hysteresis models [32], is left for future work. However, most parts of the current open-source code will be reusable also for such codes formulated in $h, h_{\max }$.

If $\mathrm{CO}_{2}$ storage becomes a large industry and sufficiently detailed models can be obtained, we believe that a fit-topurpose simulator with on-the-fly numerical calculation of all the fine-scale properties would be a useful tool to estimate the impact of the approximations made in our work to make a tractable numerical code. Although the numerical reconstruction of the vertical fluid distribution is computationally expensive, the update inside each 2D cell, which corresponds to a column of cells in the underlying 3D model, is independent of the other $2 \mathrm{D}$ cells and thus trivial to parallelize. A prototype implementation that uses GPUs to accelerate concurrent parts of the algorithm has already been developed [34]. Preliminary results obtained with this simulator indicates that assuming a sharp interface, as discussed in [8], tends to overestimate the retardation effects that structural trapping has on plume migration.

Acknowledgments The work was funded in part by Statoil ASA and the Research Council of Norway through grants no. 199878 (Numerical $\mathrm{CO}_{2}$ laboratory) and 215641 (MatMoRA-II).
Open Access This article is distributed under the terms of the Creative Commons Attribution 4.0 International License (http:// creativecommons.org/licenses/by/4.0/), which permits unrestricted use, distribution, and reproduction in any medium, provided you give appropriate credit to the original author(s) and the source, provide a link to the Creative Commons license, and indicate if changes were made.

\section{References}

1. Eiken, O., Ringrose, P., Hermanrud, C., Nazarian, B., Torp, T.A., Høier, L.: Lessons learned from 14 years of $\{\mathrm{CCS}\}$ operations: Sleipner, in salah and snøhvit. Energy Procedia 4, 5541-5548 (2011). doi:10.1016/j.egypro.2011.02.541. 10th International Conference on Greenhouse Gas Control Technologies

2. SINTEF ICT: The MATLAB reservoir simulation toolbox: numerical $\mathrm{CO}_{2}$ laboratory (2014). http://www.sintef.no/co2lab

3. The MATLAB Reservoir Simulation Toolbox: version 2015a. http://www.sintef.no/MRST/ (2015)

4. Lie, K.-A., Krogstad, S., Ligaarden, I.S., Natvig, J.R., Nilsen, H., Skaflestad, B., Open-source, M.A.T.L.A.B.: implementation of consistent discretisations on complex grids. Comput. Geosci. 16, 297-322 (2012). doi:10.1007/s10596-011-9244-4

5. Krogstad, S., Lie, K.-A., Møyner, O., Nilsen, H.M., Raynaud, X., Skaflestad B.: MRST-AD—an open-source framework for rapid prototyping and evaluation of reservoir simulation problems (2015)

6. Lie, K.-A.: An introduction to reservoir simulation using MATLAB: User guide for the Matlab reservoir simulation toolbox (MRST), SINTEF ICT. http://www.sintef.no/Projectweb/MRST/ publications. 1st Edition (2014)

7. Nilsen, H.M., Lie, K.-A., Møyner, O., Andersen, O.: Spillpoint analysis and structural trapping capacity in saline aquifers using MRST-co2lab. Computers \& Geoscience 75, 33-43 (2015). doi:10.1016/j.cageo.2014.11.002

8. Nilsen, H.M., Lie, K.-A., Andersen, O.: Robust simulation of sharp-interface models for fast estimation of $\mathrm{CO}_{2}$ trapping capacity in large-scale aquifer systems. Comput. Geosci. to appear

9. Nilsen, H.M., Lie, K.-A., Andersen, O.: Analysis of trapping capacities in the Norwegian North Sea using MRST-co2lab. Computers \& Geoscience 79, 15-26 (2015). doi:10.1016/j.cageo.2015.03.001

10. Lie, K.-A., Nilsen, H.M., Andersen, O., Møyner, O.: A simulation workflow for large-scale $\mathrm{CO}_{2}$ storage in the Norwegian North Sea, Computational Geosciences

11. Andersen, O., Lie, K.-A., Nilsen, H.M.: An open-source toolchain for simulation and optimization of aquifer-wide co2 storage, Energy Procedia

12. Halland, E.K., Mujezinović, J., Riis, F. (eds.): $\mathrm{CO}_{2}$ Storage Atlas: Norwegian Continental Shelf, Norwegian Petroleum Directorate, P.O. Box 600, NO-4003 Stavanger, Norway (2014). http://www. npd.no/en/Publications/Reports/Compiled-CO2-atlas/

13. Class, H., Ebigbo, A., Helmig, R., Dahle, H.K., Nordbotten, J.M., Celia, M.A., Audigane, P., Darcis, M., Ennis-King, J., Fan, Y., Flemisch, B., Gasda, S.E., Jin, M., Krug, S., Labregere, D., Beni, A.N., Pawar, R.J., Sbai, A., Thomas, S.G., Trenty, L., Wei, L.: A benchmark study on problems related to $\mathrm{CO} 2$ storage in geologic formations. Comput Geosci 13(4), 409-434 (2009). doi:10.1007/s10596-009-9146-X 
14. Nordbotten, J.M., Flemisch, B., Gasda, S.E., Nilsen, H.M., Fan, Y., Pickup, G.E., Wiese, B., Celia, M.A., Dahle, H.K., Eigestad, G.T., Pruess, K.: Uncertainties in practical simulation of CO2 storage. Int. J. Greenh. Gas Control. 9(0), 234-242 (2012). doi:10.1016/j.ijggc.2012.03.007

15. Nordbotten, J.M., Celia, M.A.: Geological storage of CO2: Modeling approaches for large-scale simulation. Wiley, Hoboken (2012)

16. Bear, J.: Dynamics of fluids in porous media, Dover books on physics and chemistry, Dover publications, Incorporated (1988). http://books.google.no/books?id=lurrmlFGhTEC

17. Martin, J.C.: Some mathematical aspects of two phase flow with application to flooding and gravity segregation. Prod. Monthly 22(6), 22-35 (1958)

18. Coats, K.H., Nielsen, R.L., Terune, M.H., Weber, A.G.: Simulation of three-dimensional, two-phase flow in oil and gas reservoirs. Soc. Pet. Eng. J. Dec., 377-388 (1967)

19. Martin, J.C.: Partial integration of equation of multiphase flow. Soc. Pet. Eng. J. Dec, 370-380 (1968)

20. Coats, K.H., Dempsey, J.R., Henderson, J.H.: The use of vertical equilibrium in two-dimensional simulation of three-dimensional reservoir preformance. Soc. Pet. Eng. J. Mar, 68-71 (1971)

21. Yortsos, Y.: A theoretical analysis of vertical flow equilibrium. Transp. Porous Media 18(2), 107-129 (1995). doi:10.1007/BF01064674

22. Court, B., Bandilla, K.W., Celia, M.A., Janzen, A., Dobossy, M., Nordbotten, J.M.: Applicability of vertical-equilibrium and sharp-interface assumptions in $\mathrm{CO} 2$ sequestration modeling. Int. J. Greenh. Gas Control 10, 134-147 (2012). doi:10.1016/j.ijggc.2012.04.015

23. Nilsen, H.M., Herrera, P.A., Ashraf, M., Ligaarden, I., Iding, M., Hermanrud, C., Lie, K.-A., Nordbotten, J.M., Dahle, H.K., Keilegavlen, E.: Field-case simulation of $\mathrm{CO} 2$-plume migration using vertical-equilibrium models. Energy Procedia 4(0), 3801-3808 (2011). doi:10.1016/j.egypro.2011.02.315

24. Gasda, S.E., Nordbotten, J.M., Celia, M.A.: Application of simplified models to $\mathrm{CO} 2$ migration and immobilization in large-scale geological systems. Int. J. Greenh. Gas Control 9, 72-84 (2012). doi:10.1016/j.ijggc.2012.03.001

25. Andersen, O., Gasda, S., Nilsen, H.M.: Vertically averaged equations with variable density for $\mathrm{CO}_{2}$ flow in porous media. Transp. Porous Media 107, 95-127 (2015). doi:10.1007/s11242-014-0427-z

26. Gasda, S.E., Nordbotten, J.M., Celia, M.A.: Vertically-averaged approaches to $\mathrm{CO} 2$ injection with solubility trapping. Water Resour. Res. 47, W05528 (2011). doi:10.1029/2010WR009075

27. Nordbotten, J.M., Dahle, H.K.: Impact of the capillary fringe in vertically integrated models for $\mathrm{CO}_{2}$ storage. Water Resour. Res. 47(2), W02537 (2011). doi:10.1029/2009WR008958

28. Nilsen, H.M., Syversveen, A.R., Lie, K.-A., Tveranger, J., Nordbotten, J.M.: Impact of top-surface morphology on CO2 storage capacity. Int. J. Greenh. Gas Control 11(0), 221-235 (2012). 10.1016/j.ijggc.2012.08.012

29. Gasda, S.E., Nilsen, H.M., Dahle, H.K., Gray, W.G.: Effective models for $\mathrm{CO}_{2}$ migration in geological systems with varying topography. Water Resour. Res. 48(10). doi:10.1029/2012WR012264

30. Gasda, S.E., Nilsen, H.M., Dahle, H.K.: Impact of structural heterogeneity on upscaled models for large-scale $\mathrm{CO}_{2}$ migration and trapping in saline aquifers. Adv. Water Resour. 62(Part C (0)), 520-532 (2013). doi:10.1016/j.advwatres.2013.05.003

31. Gasda, S.E., du Plessis, E., Dahle, H.K.: Upscaled models for modeling $\mathrm{CO} 2$ injection and migration in geological systems. In: Bastian, P., Kraus, J., Scheichl, R., Wheeler, M. (eds.) Simulation of Flow in Porous Media, Vol. 12 of Radon Series on Computational and Applied Mathematics, De Gruyter, pp. 1-38. Berlin, Boston (2013). doi:10.1515/9783110282245.1

32. Doster, F., Nordbotten, J.M., Celia, M.A.: Hysteretic upscaled constitutive relationships for vertically integrated porous media flow. Comput. Visual. Sci. 15, 147-161 (2012). 10.1007/s00791013-0206-3

33. Ligaarden, I.S., Nilsen, H.M.: Numerical aspects of using vertical equilibrium models for simulating $\mathrm{CO}_{2}$ sequestration. In: Proceedings of ECMOR XII-12th European Conference on the Mathematics of Oil Recovery, EAGE, Oxford, UK, p. 2010

34. Seternes, G.: Simulations of co2 migration with a fully-integrated ve model on the gpu, Master's thesis, Norwegian University of Science and Technology. https://daim.idi.ntnu.no/masteroppgave? id=12404 (2015)

35. Singh, V., Cavanagh, A., Hansen, H., Nazarian, B., Iding, M., Ringrose, P.: Reservoir modeling of $\mathrm{CO}_{2}$ plume behavior calibrated against monitoring data from Sleipner, Norway. In: SPE Annual Technical Conference and Exhibition, 1922 September 2010, Florence, Italy, 2010, SPE 134891-MS. doi:10.2118/134891-MS

36. Bandilla, K.W., Celia, M.A., Leister, E.: Impact of model complexity on $\mathrm{CO}_{2}$ plume modeling at sleipner. Energy Procedia 63, 3405-3415 (2014). doi:10.1016/j.egypro.2014.11.369. 12th International Conference on Greenhouse Gas Control Technologies, GHGT-12

37. Gasda, S.E., Nordbotten, J.M., Celia, M.A.: Vertical equilibrium with sub-scale analytical methods for geological C02 sequestration. Comput. Geosci 13(4), 469-481 (2009). doi:10.1007/s10596-009-9138-x

38. Andersen, O., Nilsen, H.M., Lie, K.-A.: Reexamining $\mathrm{CO}_{2}$ storage capacity and utilization of the Utsira Formation. In: ECMOR XIV $-14^{\text {th }}$ European Conference on the Mathematics of Oil Recovery, Catania, Sicily, Italy, 8-11 September 2014, EAGE (2014). doi:10.3997/2214-4609.20141809

39. Lie, K.-A., Nilsen, H.M., Andersen, O., Møyner, O.: A simulation workflow for large-scale $\mathrm{CO}_{2}$ storage in the Norwegian North Sea. In: ECMOR XIV- $14^{\text {th }}$ European Conference on the Mathematics of Oil Recovery, Catania, Sicily, Italy, 8-11 September 2014, EAGE (2014). doi:10.3997/2214-4609.20141877

40. Juanes, R., Spiteri, E.J., Orr Jr., F.M., Blunt, M.J.: Impact of relative permeability hysteresis on geological $\mathrm{CO}_{2}$ storage. Water Resour. Res. 42, W12418. doi:10.1029/2005WR004806

41. Span, R., Wagner, W.: A new equation of state for carbon dioxide covering the fluid region from triple-point temperature to $1100 \mathrm{~K}$ at pressures up to $800 \mathrm{MPa}$. J. Phys. Chem. Ref. Data 25(6), 15091597 (1996) 